\title{
COMMUTING DIAGRAMS FOR THE TNT ELEMENTS ON CUBES
}

\author{
BERNARDO COCKBURN AND WEIFENG QIU
}

\begin{abstract}
We present commuting diagrams for the de Rham complex for new elements defined on cubes which use tensor product spaces. The distinctive feature of these elements is that, in sharp contrast with previously known results, they have the TiNiest spaces containing Tensor product spaces of polynomials of degree $k$, hence their acronym TNT. In fact, the local spaces of the TNT elements differ from the standard tensor product spaces by spaces whose dimension is a small number independent of the degree $k$. Such a number is 7 (the number of vertices of the cube minus one) for the space associated with the divergence operator, 18 (the number of faces of the cube times the number of vertices of a face minus one) for the space associated with the curl operator, and 12 (the number of edges of the cube times the number of vertices of an edge minus one) for the space associated with the gradient operator.
\end{abstract}

\section{INTRODUCTION}

In this paper, we present a new commuting diagram of the form

$$
\begin{aligned}
& H^{r}(\Omega) \stackrel{\nabla}{\longrightarrow} H^{r-1}(\operatorname{curl}, \Omega) \stackrel{\nabla \times}{\longrightarrow} H^{r-1}(\operatorname{div}, \Omega) \stackrel{\nabla \cdot}{\longrightarrow} H^{r-1}(\Omega) \\
& \downarrow \Pi_{H_{h}} \quad \downarrow \Pi_{E_{h}} \quad \downarrow \Pi_{V_{h}} \quad \downarrow \Pi_{W_{h}} \\
& H\left(\Omega_{h}\right) \quad \stackrel{\nabla}{\longrightarrow} \quad E\left(\Omega_{h}\right) \quad \stackrel{\nabla \times}{\longrightarrow} \quad V\left(\Omega_{h}\right) \quad \stackrel{\nabla \cdot}{\longrightarrow} W\left(\Omega_{h}\right)
\end{aligned}
$$

where we take $r>3 / 2$ to ensure that the projections are well defined. Here, $\Omega$ is an open subset of $\mathbb{R}^{3}$ which accepts triangulations $\Omega_{h}:=\{K\}$ where the elements $K$ are suitably defined linear or nonlinear mappings of a cubic reference element $\widehat{K}$. In other words, we construct finite-dimensional spaces and projections satisfying the commutativity properties

$$
\begin{array}{rlr}
\nabla \cdot \Pi_{V_{h}}=\Pi_{W_{h}} \nabla \cdot & & \text { on } H^{r-1}(\operatorname{div}, \Omega), \\
\nabla \times \Pi_{E_{h}}=\Pi_{V_{h}} \nabla \times & & \text { on } H^{r-1}(\operatorname{curl}, \Omega), \\
\nabla \Pi_{H_{h}}=\Pi_{E_{h}} \nabla & & \text { on } H^{r}(\Omega) .
\end{array}
$$

Received by the editor July 12, 2011 and, in revised form, March 5, 2012, March 30, 2012, and June 21, 2012.

2010 Mathematics Subject Classification. Primary 65N30, 65L12.

Key words and phrases. Commuting diagrams, cubic element, tensor product spaces.

The first author was partially supported by the National Science Foundation (Grant DMS0712955) and by the Minnesota Supercomputing Institute.

The second author gratefully acknowledges the collaboration opportunities provided by IMA (Minneapolis) during their 2010-2012 program.

Corresponding author: Weifeng Qiu. 
The novelty of our result lies in the fact that the above spaces are constructed by using elements that are the TiNiest spaces containing the standard Tensor product spaces of polynomials of degree $k \geq 1$; this is why we call them the TNT elements. The main motivation for considering these methods is that their tensor-product structure can be exploited to achieve a very efficient implementation; see [14, 13, 6] and the references therein. On the reference cube, $\widehat{K}$, the spaces of the TNT elements differ from the above-mentioned tensor product spaces by spaces whose dimension is a small number independent of the degree $k$. Indeed, we have

$$
\begin{array}{rlrl}
W(\widehat{K}) & =Q_{k}(\widehat{K}), & \\
V(\widehat{K}) & =\left[Q_{k}(\widehat{K}) \times Q_{k}(\widehat{K}) \times Q_{k}(\widehat{K})\right] \oplus \delta_{V}(\widehat{K}), & & \operatorname{dim} \delta_{V}(\widehat{K})=7, \\
E(\widehat{K}) & =\left[Q_{k}(\widehat{K}) \times Q_{k}(\widehat{K}) \times Q_{k}(\widehat{K})\right] \oplus \delta_{E}(\widehat{K}), & \operatorname{dim} \delta_{E}(\widehat{K})=18, \\
H(\widehat{K})=Q_{k}(\widehat{K}) \oplus \delta_{H}(\widehat{K}), & & \operatorname{dim} \delta_{H}(\widehat{K})=12,
\end{array}
$$

where $Q_{k}(\widehat{K}):=P_{k, k, k}(\widehat{K})$ and $P_{\ell_{1}, \ell_{2}, \ell_{3}}(\widehat{K})$ denotes the space of tensor product polynomials of degree at most $\ell_{i}$ in the $i$-th variable, $i=1,2,3$.

The dimension of the spaces $\delta_{V}(\widehat{K}), \delta_{E}(\widehat{K})$ and $\delta_{H}(\widehat{K})$ seems to be strongly related to the properties of the divergence operator on the tensor product spaces of three, two and one dimensions, respectively. Moreover, it has a precise geometric interpretation. Indeed, $\operatorname{dim} \delta_{V}(\widehat{K})=7$ is the number of vertices of a cube minus one, $\operatorname{dim} \delta_{E}(\widehat{K})=18$ is the number of faces of a cube times the number of vertices of a face minus one, and $\operatorname{dim} \delta_{H}(\widehat{K})=12$ is the number of edges times the number of vertices of an edge minus one.

It is interesting to compare this commuting diagram to the only other commuting diagram for elements defined on cubes using tensor product spaces. It was found back in 1980, [10, and uses the following local spaces:

$$
\begin{aligned}
W(\widehat{K}) & =\mathcal{P}_{k, k, k}(\widehat{K}), \\
V(\widehat{K}) & =\mathcal{P}_{k+1, k, k}(\widehat{K}) \times \mathcal{P}_{k, k+1, k}(\widehat{K}) \times \mathcal{P}_{k, k, k+1}(\widehat{K}), \\
E(\widehat{K}) & =\mathcal{P}_{k, k+1, k+1}(\widehat{K}) \times \mathcal{P}_{k+1, k, k+1}(\widehat{K}) \times \mathcal{P}_{k+1, k+1, k}(\widehat{K}), \\
H(\widehat{K}) & =\mathcal{P}_{k+1, k+1, k+1}(\widehat{K}),
\end{aligned}
$$

or, using our notation to facilitate a comparison of the dimension of the spaces,

$$
\begin{array}{rlrl}
W(\widehat{K}) & =Q_{k}(\widehat{K}), & \\
V(\widehat{K}) & =\left[Q_{k}(\widehat{K}) \times Q_{k}(\widehat{K}) \times Q_{k}(\widehat{K})\right] \oplus \delta_{V}(\widehat{K}), & & \operatorname{dim} \delta_{V}(\widehat{K})=3 k^{2}+6 k+3, \\
E(\widehat{K}) & =\left[Q_{k}(\widehat{K}) \times Q_{k}(\widehat{K}) \times Q_{k}(\widehat{K})\right] \oplus \delta_{E}(\widehat{K}), & & \operatorname{dim} \delta_{E}(\widehat{K})=6 k^{2}+15 k+9, \\
H(\widehat{K}) & =Q_{k}(\widehat{K}) \oplus \delta_{H}(\widehat{K}), & & \operatorname{dim} \delta_{H}(\widehat{K})=3 k^{2}+9 k+7 .
\end{array}
$$

There is a relatively recent, renewed interest in the use of commuting diagrams for the design and analysis of finite element methods; see [2, 3] and the references therein. Most known commuting diagrams use simplexes as elements. Examples are the commuting diagram obtained in [10] for tetrahedral meshes, its generalization to arbitrary-dimensional simplexes in [2], and its extension to variable-degree elements in [11. Most commuting diagrams using rectangular or cubic elements do not use tensor product spaces. In fact, the only commuting diagram of that type is the one found in [10], as was mentioned above. 
We did not find the TNT elements by trying to understand if it was possible to reduce the size of the tensor product spaces used in [10. We found them by pursuing a quite different goal. Indeed, it was during the systematic construction of superconvergent hybridizable discontinuous Galerkin methods for diffusion problems [8] that the TNT elements corresponding to the commuting diagram associated with the divergence operator were uncovered. In this paper, we provide a different derivation of such a diagram and then complete the diagrams associated with the curl operator and then the diagram associated with the gradient operator.

The paper is organized as follows. In Section 2, we state our main results for the reference element, and in Section 3, we extend them for suitably defined triangulations. In Section 4, we provide detailed proofs of the results on the reference element and in Section 5, of the results concerning the triangulations. We end in Section 6 by describing a couple of simple applications, by arguing in what sense the dimension of the spaces $\delta_{V}(K), \delta_{E}(K)$ and $\delta_{H}(K)$ is the smallest possible, and, for the sake of completeness, by briefly describing the two-dimensional case.

\section{The MAin RESUlts}

2.1. Commuting diagrams for the reference cube $\widehat{K}$. Here, we restrict ourselves to the study of the commuting diagrams for the case in which $\Omega_{h}$ is nothing but the unit cube $\widehat{K}:=(0,1)^{3}$.

We proceed as follows. We begin by introducing the notation and conventions we are going to use. Then, we introduce the local spaces and define the corresponding projections. Finally, we state and briefly discuss our main result, Theorem 2.6. which describes the commutativity properties of the projections.

To simplify the notation, we omit the "hat" typically used to identify objects associated to the reference element whenever there is no possible confusion. Thus, to denote the reference unit cube $\widehat{K}$, we simply write $K$.

\subsubsection{Notation and conventions.}

Geometric objects. We begin by defining various geometric objects associated with the element $K$.

We denote by $\mathcal{F}(K)$ the set of faces $f$ of the cube $K$. For any face $f$, we denote by $n_{f}$ or, when there is no confusion, simply by $n$, the outward unit normal of $K$ on the face $f$. We also denote by $\mathcal{E}(K)$ the set of edges $e$ of the cube $K$. For any edge $e$ of the cube $K$, we denote by $t_{e}$ or, when there is not confusion, simply by $t$, a unit vector tangent to the edge $e$. We also denote by $\mathcal{E}(f)$ the set of edges $e$ of the face $f$. For any edge $e$, we denote by $n_{e}$ or, when there is no confusion, simply by $n$, the outward unit normal of $f$ on the edge $e$, which is perpendicular to $e$ and tangential to $f$. Finally, we denote by $\mathcal{V}(K)$ the set of vertices $v$ of the cube $K$, by $\mathcal{V}(f)$ the set of vertices $v$ of the face $f$, and by $\mathcal{V}(e)$ the set of vertices $v$ of the edge $e$.

We are also going to need the following notation. We denote by $\left\{e_{i}\right\}_{i=1}^{3}$ the canonical basis of $\mathbb{R}^{3}$. Then, given the point $v_{C} \in \mathbb{R}^{3}, d \leq 3$, and the monotone increasing injective function $\sigma:\{1, \ldots, d\} \mapsto\{1,2,3\}$, we set

$$
C:=v_{C}+\left\{\sum_{j=1}^{d} x_{\sigma(j)} e_{\sigma(j)}: x_{\sigma(j)} \in[0,1] \text { for } j=1, \ldots, d\right\} .
$$


In other words, $C$ is a $d$-dimensional cube embedded in $\mathbb{R}^{3}$. For example, $C$ can be the cube $K(d=3)$, any of its faces $f(d=2)$, or any of its edges $e(d=1)$.

Spaces. To the $d$-dimensional cube $C$, we associate the standard tensor product spaces

$$
Q_{k}(C):=\bigotimes_{j=1}^{d} P_{k}\left(x_{\sigma(j)}\right) \quad \text { and } \quad\left[Q_{k}(C)\right]^{d}:=\sum_{j=1}^{d} Q_{k}(C) e_{\sigma(j)},
$$

as well as a key, auxiliary space, $D_{k}(C)$, defined by

$$
D_{k}(C):=\operatorname{span}\left\{v_{k}\left(\sum_{j=1}^{d} \ell_{j} x_{\sigma(j)} e_{\sigma(j)}\right): \ell_{1}, \ldots, \ell_{d} \in\{0,1\}, \ell_{1}+\cdots+\ell_{d} \neq 0\right\},
$$

where the $i$-th component of the function $v_{k}\left(x_{1}, x_{2}, x_{3}\right)$ is

$$
\begin{array}{ll}
\tilde{B}_{k+1}\left(x_{1}\right) \tilde{P}_{k}\left(x_{2}\right) \tilde{P}_{k}\left(x_{3}\right) & \text { if } i=1, \\
\tilde{P}_{k}\left(x_{1}\right) \tilde{B}_{k+1}\left(x_{2}\right) \tilde{P}_{k}\left(x_{3}\right) & \text { if } i=2, \\
\tilde{P}_{k}\left(x_{1}\right) \tilde{P}_{k}\left(x_{2}\right) \tilde{B}_{k+1}\left(x_{3}\right) & \text { if } i=3 .
\end{array}
$$

Here, $\tilde{P}_{\ell}$ is the so-called shifted Legendre polynomial on $[0,1]$ of degree $\ell \geq 0$; that is, $\tilde{P}_{\ell}(y):=P_{\ell}(-1+2 y)$, where $P_{\ell}$ is the Legendre polynomial of degree $\ell$. The function $\tilde{B}_{\ell}$ is defined as follows:

$$
\tilde{B}_{\ell+1}:= \begin{cases}0 & \text { if } \ell=0, \\ \left(\tilde{P}_{\ell+1}-\tilde{P}_{\ell-1}\right) /(4 \ell+2) & \text { if } \ell>0 .\end{cases}
$$

There are two properties of this function which are important in our analysis. The first is that

$$
\tilde{B}_{\ell+1}^{\prime}=\tilde{P}_{\ell} \quad \text { for } \ell>0 .
$$

This can be easily verified by using standard properties of the Legendre polynomials. The second is that

$$
\tilde{B}_{\ell+1}(x)=0 \quad \text { for } x \in\{0,1\},
$$

since $\tilde{P}_{\ell}(1)=P_{\ell}(1)=1$ and $\tilde{P}_{\ell}(0)=P_{\ell}(-1)=(-1)^{\ell}$. Thanks to this property, the functions $\tilde{B}_{\ell+1}$ are called bubble functions. Illustrations of the spaces $D_{k}(C)$ are considered in the next subsection.

To conclude, we introduce two special functions. To each face $f \in \mathcal{F}(K)$, we associate the linear function $\lambda_{f}$ defined to be equal to one on the face $f$ and equal to zero on the other parallel face of $K$. To each edge $e \in \mathcal{E}(K)$, we associate the bilinear function $\lambda_{e}$ defined to be equal to one on the edge $e$ and equal to zero on the other three parallel edges of $K$.

Extensions, traces, differential operators. If $\phi$ is a function defined on the face $f$, we extend it to the cube $K$ by keeping it constant along the lines perpendicular to the face $f$. Similarly, if $\varphi$ is a function defined on the edge $e$, we extend it to the cube $K$ by keeping it constant along the planes perpendicular to the edge $e$. To simplify the notation, the extensions of $\phi$ and $\varphi$ will be denoted simply by $\phi$ and $\varphi$, respectively. In this way, we make no explicit distinction between, for example, the space $\left[Q_{k}(f)\right]^{2}$ and the space of their extensions to $K$ which we also denote by $\left[Q_{k}(f)\right]^{2}$. 
If $q$ is a vector-valued function defined on $K$, the trace of its normal component $q \cdot n_{f}$ on the face $f$ is denoted by $t r_{n, f} q$, or simply by $t r_{n} q$ when there is no confusion. We define the trace of the tangential component of $q$ on the face

$$
f:=v_{f}+\left\{\sum_{j=1}^{2} x_{\sigma(j)} e_{\sigma(j)}: x_{\sigma(j)} \in[0,1] \text { for } j=1,2\right\}
$$

to be

$$
\operatorname{tr}_{t, f} q=\left(q_{\sigma(1)}, q_{\sigma(2)}\right)^{\top} \in \mathbb{R}^{2} .
$$

When there is no confusion, we use simply by $t r_{t} q$. If $q$ is a vector-valued function defined on $f$, the trace of its normal component $q \cdot n_{e}$ on the edge $e$ is denoted by $t r_{n, e} q$, or simply by $t r_{n} q$ when there is no confusion. If $u$ is a scalar-valued function defined on $C$, its trace on the border of $C, \partial C$, is denoted by $\operatorname{tr}_{\partial C} u$, or simply by $\operatorname{tr} u$ when there is no confusion.

Finally, for a vector-valued function $q$ and a scalar-valued function $u$ defined on the cube $K$, we define the operators

$$
\begin{aligned}
\nabla_{f} \cdot q & :=\left(n_{f} \times \nabla\right) \cdot\left(n_{f} \times q\right), & & \nabla_{f} \times u:=-\left(n_{f} \times \nabla\right) u, \\
\nabla_{f} u & :=n_{f} \times \nabla u \times n_{f}, & & \nabla_{e} u:=t_{e} \cdot \nabla u .
\end{aligned}
$$

2.1.2. Definition of the spaces. We are going to use the following spaces.

Definition 2.1. We take

$$
\begin{aligned}
W(K) & :=Q_{k}(K), \\
V(K) & :=\left[Q_{k}(K)\right]^{3} \oplus \delta_{V}(K), \quad \delta_{V}(K):=D_{k}(K), \\
E(K) & :=\left[Q_{k}(K)\right]^{3} \oplus \delta_{E}(K), \quad \delta_{E}(K):=\bigoplus_{f \in \mathcal{F}(K)} \lambda_{f} n_{f} \times D_{k}(f), \\
H(K) & :=Q_{k}(K) \oplus \delta_{H}(K), \quad \delta_{H}(K):=\bigoplus_{e \in \mathcal{E}(K)} \lambda_{e} t_{e} \cdot D_{k}(e) .
\end{aligned}
$$

The spaces $\delta_{V}(K), \delta_{E}(K)$ and $\delta_{H}(K)$ can be thought of as being generated by special bubble functions. Next, we argue why.

The space $\delta_{V}(K)$. By the definition of the space $D_{k}(K)$, (2.1), we have

$$
\begin{aligned}
& \delta_{V}(K)=\operatorname{span}\{ v_{k}\left(x_{1}, 0,0\right), v_{k}\left(0, x_{2}, 0\right), v_{k}\left(0,0, x_{3}\right), \\
& v_{k}\left(0, x_{2}, x_{3}\right), v_{k}\left(x_{1}, 0, x_{3}\right), v_{k}\left(x_{1}, x_{2}, 0\right), \\
&\left.v_{k}\left(x_{1}, x_{2}, x_{3}\right)\right\} \\
&=\operatorname{span}\left\{\left(\tilde{B}_{k+1}\left(x_{1}\right), 0,0\right),\left(0, \tilde{B}_{k+1}\left(x_{2}\right), 0\right),\left(0,0, \tilde{B}_{k+1}\left(x_{3}\right)\right),\right. \\
&\left(0, \tilde{B}_{k+1}\left(x_{2}\right) \tilde{P}_{k}\left(x_{3}\right), \tilde{P}_{k}\left(x_{2}\right) \tilde{B}_{k+1}\left(x_{3}\right)\right), \\
&\left(\tilde{B}_{k+1}\left(x_{1}\right) \tilde{P}_{k}\left(x_{3}\right), 0, \tilde{P}_{k}\left(x_{1}\right) \tilde{B}_{k+1}\left(x_{3}\right)\right), \\
&\left(\tilde{B}_{k+1}\left(x_{1}\right) \tilde{P}_{k}\left(x_{2}\right), \tilde{P}_{k}\left(x_{1}\right) \tilde{B}_{k+1}\left(x_{2}\right), 0\right), \\
&\left.\left(\tilde{B}_{k+1}\left(x_{1}\right) \tilde{P}_{k}\left(x_{2}\right) \tilde{P}_{k}\left(x_{3}\right), \tilde{P}_{k}\left(x_{1}\right) \tilde{B}_{k+1}\left(x_{2}\right) \tilde{P}_{k}\left(x_{3}\right), \tilde{P}_{k}\left(x_{1}\right) \tilde{P}_{k}\left(x_{2}\right) \tilde{B}_{k+1}\left(x_{3}\right)\right)\right\} .
\end{aligned}
$$

By property (2.2C), $\tilde{B}_{k+1}(0)=\tilde{B}_{k+1}(1)=0$, and we see that each of the functions generating the space $\delta_{V}(K)$ has normal trace equal to zero. So, we can think of them as some kind of vector-valued bubble functions associated to the cube $K$; see Figure 1 

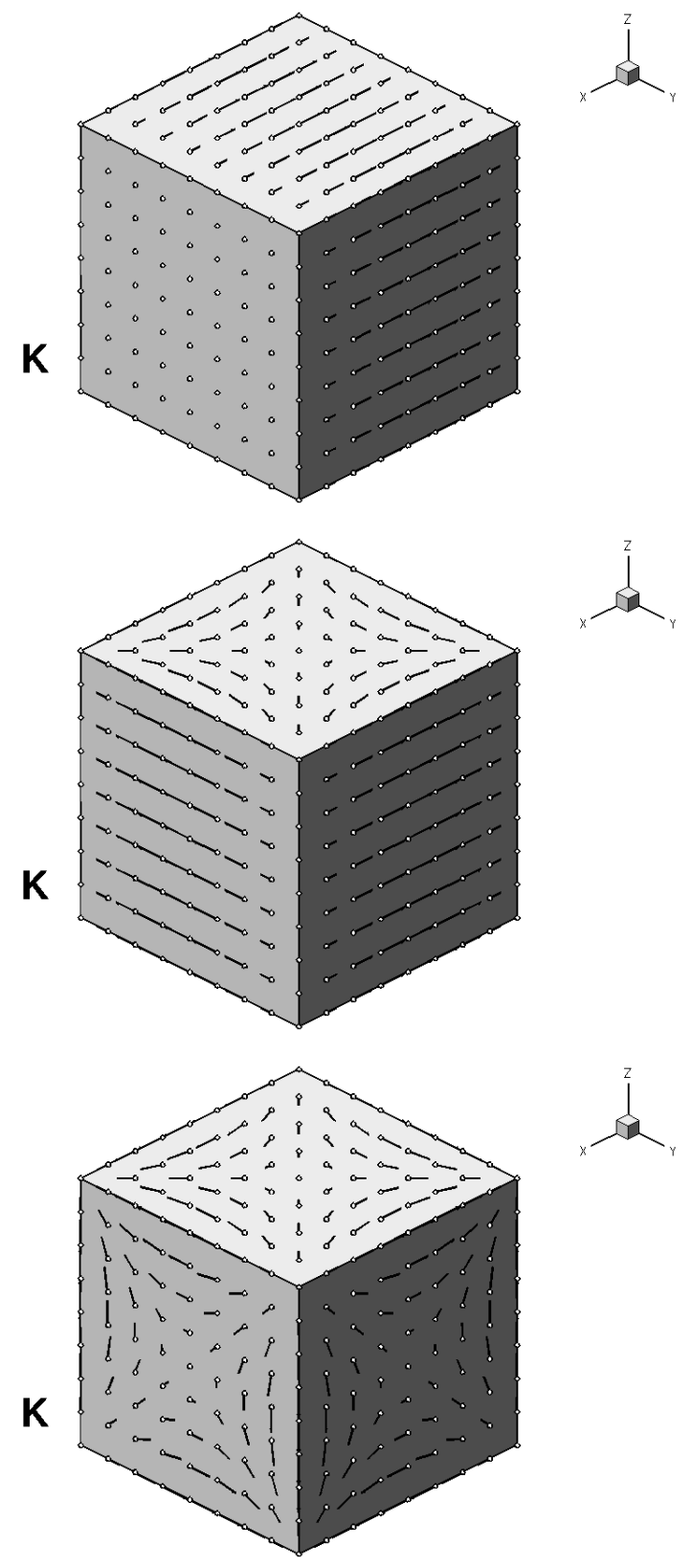

Figure 1. Trace of the bubble functions spanning the space $\delta_{V}(K)$ : The functions $v_{k}\left(x_{1}, 0,0\right)$ (top), $v_{k}\left(x_{1}, x_{2}, 0\right)$ (middle), and $v_{k}\left(x_{1}, x_{2}, x_{3}\right)$ (bottom). Note that their normal trace is zero. The plot of the functions $v_{k}\left(0, x_{2}, 0\right)$ and $v_{k}\left(0,0, x_{3}\right)$ is obtained by suitably rotating the plot of $v_{k}\left(x_{1}, 0,0\right)$. Similarly, the plot of the functions $v_{k}\left(x_{1}, 0, x_{2}\right)$ and $v_{k}\left(0, x_{2}, x_{3}\right)$ are obtained by suitably rotating the plot of $v_{k}\left(x_{1}, x_{2}, 0\right)$. Recall that the dimension of $\delta_{V}(K)$ is 7 . Here $k=1$ and $(x, y, z):=\left(x_{1}, x_{2}, x_{3}\right)$. 

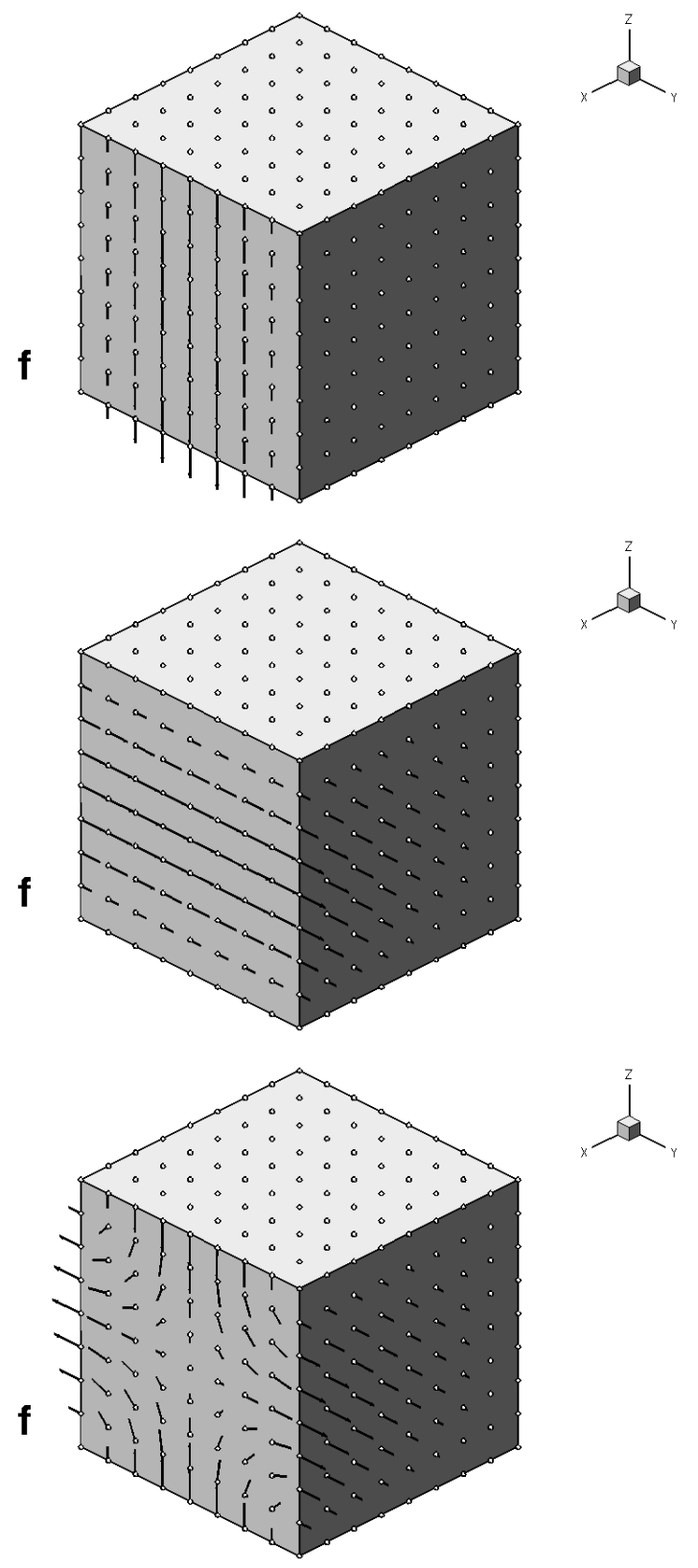

Figure 2. Trace of the bubble functions spanning the space $\delta_{E}(K)$ : The functions associated to the face $f$ lying on the plane $x_{1}=1$ are $\lambda_{f} n_{f} \times v_{k}\left(0, x_{2}, 0\right)$ (left), $\lambda_{f} n_{f} \times v_{k}\left(0,0, x_{3}\right)$ (middle), and $\lambda_{f} n_{f} \times v_{k}\left(0, x_{2}, x_{3}\right)$ (bottom). Here $\lambda_{f}=x_{1}$ and $n_{f}=(1,0,0)$. Note that their tangential trace is zero outside the face $f$. For each face of the cube there are three spanning functions of the space $\delta_{E}(K)$ so that its dimension is $18=(6$ faces $) \times 3$. Here $k=1$ and $(x, y, z):=\left(x_{1}, x_{2}, x_{3}\right)$. 
The space $\delta_{E}(K)$. To illustrate what this space looks like, we only need to display the space $\lambda_{f} n_{f} \times D_{k}(f)$ for any of the faces $f$ of $K$. For example, for the face $f$ lying on the plane $x_{1}=1$, we have that $\lambda_{f}=x_{1}, n_{f}=(1,0,0)$ and, by the definition of the space $D_{k}(f)$, 2.1),

$$
\begin{aligned}
& D_{k}(f)= \operatorname{span}\left\{v_{k}\left(0, x_{2}, 0\right), v_{k}\left(0,0, x_{3}\right), v_{k}\left(0, x_{2}, x_{3}\right)\right\} \\
&=\operatorname{span}\left\{\left(0, \tilde{B}_{k+1}\left(x_{2}\right), 0\right),\left(0,0, \tilde{B}_{k+1}\left(x_{3}\right)\right),\right. \\
&\left.\left(0, \tilde{B}_{k+1}\left(x_{2}\right) \tilde{P}_{k}\left(x_{3}\right), \tilde{P}_{k}\left(x_{2}\right) \tilde{B}_{k+1}\left(x_{3}\right)\right)\right\} .
\end{aligned}
$$

As a consequence,

$$
\begin{aligned}
\lambda_{f} n_{f} \times D_{k}(f)=\operatorname{span}\{ & \left(0,0, x_{1} \tilde{B}_{k+1}\left(x_{2}\right)\right),\left(0,-x_{1} \tilde{B}_{k+1}\left(x_{3}\right), 0\right), \\
& \left.\left(0,-x_{1} \tilde{P}_{k}\left(x_{2}\right) \tilde{B}_{k+1}\left(x_{3}\right), x_{1} \tilde{B}_{k+1}\left(x_{2}\right) \tilde{P}_{k}\left(x_{3}\right)\right)\right\} .
\end{aligned}
$$

Note that, again by property (2.2C) of the bubble functions $\tilde{B}_{k+1}$, the tangential trace of each of the functions generating this space is zero, except on the face $f$; see an illustration in Figure 2. Because of this, we can then think of these functions as vector-valued bubble functions associated to the face $f$.

The space $\delta_{H}(K)$. To illustrate what this space looks like, we only need to display the space $\lambda_{e} t_{e} \cdot D_{k}(e)$ for any of the edges $e$ of $K$. For example, for the edge $e$ lying on the line $x_{1}=x_{2}=1$, we have that $\lambda_{e}=x_{1} x_{2}, t_{e}=(0,0,1)$ and, by the definition of the space $D_{k}(e)$, (2.1),

$$
D_{k}(e)=\operatorname{span}\left\{v_{k}\left(0,0, x_{3}\right)\right\}=\operatorname{span}\left\{\left(0,0, \tilde{B}_{k+1}\left(x_{3}\right)\right)\right\} .
$$

As a consequence, we have

$$
\lambda_{e} t_{e} \cdot D_{k}(e)=\operatorname{span}\left\{x_{1} x_{2} \tilde{B}_{k+1}\left(x_{3}\right)\right\} .
$$

Note that the trace of the function generating this space is zero except on the two faces sharing the edge $e$; see an illustration in Figure 3. We can then think of this function as a scalar-valued bubble function associated to the edge $e$.

2.1.3. Definition of the projections. In what follows, $(\cdot, \cdot)_{C}$ denotes the standard $L^{2}(C)$-inner products. We are ready to define the projections.

Definition 2.2. The operator $\Pi_{W}: L^{2}(K) \rightarrow W(K)$ is given by

$$
\left(\Pi_{W} u-u, w\right)_{K}=0 \quad \forall w \in Q_{k}(K) .
$$

Definition 2.3. The operator $\Pi_{V}: H^{r-1}(\operatorname{div}, K) \rightarrow V(K)$ is given by

$$
\begin{aligned}
\left(\left(\Pi_{V} q-q\right) \cdot n, \mu\right)_{f}=0 & \forall \mu \in Q_{k}(f) \forall f \in \mathcal{F}(K), \\
\left(\Pi_{V} q-q, \nabla w\right)_{K}=0 & \forall w \in Q_{k}(K), \\
\left(\Pi_{V} q-q, v\right)_{K}=0 & \forall v \in \nabla \times \operatorname{Ker}\left(\left.t r_{t}\right|_{\left[Q_{k}(K)\right]^{3}}\right) .
\end{aligned}
$$

Definition 2.4. The operator $\Pi_{E}: H^{r-1}(\operatorname{curl}, K) \rightarrow E(K)$ is given by

$$
\begin{aligned}
\left(\left(\Pi_{E} \omega-\omega\right) \cdot t, \mu\right)_{e}=0 & \forall \mu \in Q_{k}(e) \forall e \in \mathcal{E}(K), \\
\left(\left(\Pi_{E} \omega-\omega\right) \times n, \nabla \zeta\right)_{f}=0 & \forall \zeta \in Q_{k}(f) \quad \forall f \in \mathcal{F}(K), \\
\left(\left(\Pi_{E} \omega-\omega\right) \times n, z\right)_{f}=0 & \forall z \in \nabla_{f} \times \operatorname{Ker}\left(\left.\operatorname{tr}\right|_{Q_{k}(f)}\right) \forall f \in \mathcal{F}(K), \\
\left(\nabla \times\left(\Pi_{E} \omega-\omega\right), \nabla \times v\right)_{K}=0 & \forall v \in \operatorname{Ker}\left(\left.\operatorname{tr}_{t}\right|_{\left.Q_{k}(K)\right]^{3}}\right), \\
\left(\Pi_{E} \omega-\omega, v\right)_{K}=0 & \forall v \in \nabla \operatorname{Ker}\left(\left.\operatorname{tr}\right|_{Q_{k}(K)}\right) .
\end{aligned}
$$




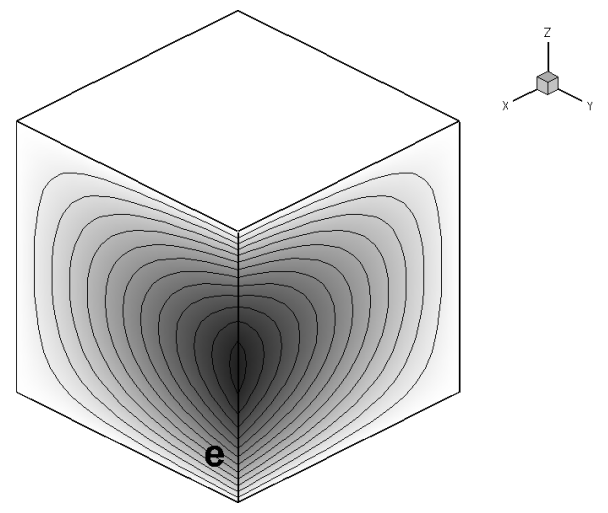

Figure 3. Trace of the bubble functions spanning the space $\delta_{H}(K)$ : The function associated with the edge $e$ lying on the line $x_{1}=x_{2}=1$ is $\lambda_{e} t_{e} \cdot v_{k}\left(0,0, x_{3}\right)$. Here $\lambda_{e}=x_{1} x_{2}$ and $t_{e}=(0,0,1)$. Note also that it is zero (white) on the faces not sharing the edge $e$. For each edge of the cube, there is one of these functions so that the dimension of $\delta_{H}(K)$ is $12=(12$ faces $) \times 1$. Here $k=1$ and $(x, y, z):=\left(x_{1}, x_{2}, x_{3}\right)$.

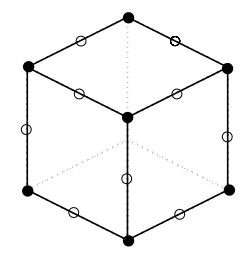

d.o.f. $\Pi_{H}=12+8=20$

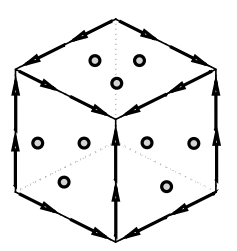

d.o.f. $\Pi_{E}=2 \times 12+3 \times 6=42$

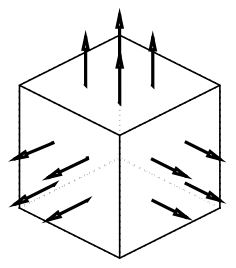

d.o.f. $\Pi_{V}=4 \times 6+7=31$

Figure 4. Degrees of freedom for the case $k=1: \Pi_{H}$ (top, left), $\Pi_{E}$ (top, right) and $\Pi_{V}$ (bottom). There are 7 degrees of freedom for $\Pi_{V}$ which are not represented here; they are associated to the interior of the cube $K$.

Definition 2.5. The operator $\Pi_{H}: H^{r}(K) \rightarrow H(K)$ is given by

$$
\begin{aligned}
\left(\Pi_{H} u-u\right)(v)=0 & \forall v \in \mathcal{V}(K), \\
\left(\Pi_{H} u-u, \nabla_{e} \mu\right)_{e}=0 & \forall \mu \in Q_{k}(e) \quad \forall e \in \mathcal{E}(K), \\
\left(\Pi_{H} u-u, \Delta \zeta\right)_{f}=0 & \forall \zeta \in \operatorname{Ker}\left(\left.\operatorname{tr}\right|_{Q_{k}(f)}\right) \quad \forall f \in \mathcal{F}(K), \\
\left(\nabla\left(\Pi_{H} u-u\right), \nabla w\right)_{K}=0 & \forall w \in \operatorname{Ker}\left(\left.\operatorname{tr}\right|_{Q_{k}(K)}\right) .
\end{aligned}
$$

As an illustration, we provide, in Figure 4, a traditional pictorial representation of the degrees of freedom of the above-defined projections for the smallest spaces, that is, for the case $k=1$. Note that in this simple case, the degrees of freedom of 
the projections $\Pi_{H}$ are associated with the points and the edges of the cube, those of $\Pi_{E}$ are associated with the edges and the faces of the cube, and those of $\Pi_{V}$ are associated with the faces of the cube and the cube itself (not represented).

2.1.4. The commuting diagrams. We are now ready to state our result.

Theorem 2.6. For any polynomial degree $k \geq 1$, the operators $\Pi_{V}, \Pi_{E}$ and $\Pi_{H}$ are well-defined projections. Moreover, we have that

$$
\begin{aligned}
\Pi_{W} \nabla \cdot q & =\nabla \cdot \Pi_{V} q & & \forall q \in H^{1}\left(K, \mathbb{R}^{3}\right), \\
\Pi_{V} \nabla \times w & =\nabla \times \Pi_{E} w & & \forall w \in H^{1}\left(K, \mathbb{R}^{3}\right), \\
\Pi_{E} \nabla u & =\nabla \Pi_{H} u & & \forall u \in H^{1}(K, \mathbb{R}) .
\end{aligned}
$$

Perhaps the most salient feature of this result is that it holds for spaces $\delta_{V}(K)$, $\delta_{E}(K)$ and $\delta_{H}(K)$ whose dimension is independent of the polynomial degree $k$. Indeed, a simple computation gives that

$$
\begin{aligned}
& \operatorname{dim} \delta_{V}(K)=\operatorname{dim} D_{k}(K)=7, \\
& \operatorname{dim} \delta_{E}(K)=(6 \text { faces }) \times \operatorname{dim} D_{k}(f)=6 \times 3, \\
& \operatorname{dim} \delta_{H}(K)=(12 \text { edges }) \times \operatorname{dim} D_{k}(e)=12 \times 1 .
\end{aligned}
$$

2.2. Commuting diagrams for the entire mesh $\Omega_{h}$. We are now ready to state our results for the entire mesh $\Omega_{h}$. We begin by

\subsubsection{Definition of the meshes.}

Definition 2.7. A closed set $K \subset \mathbb{R}^{3}$ is called a generalized hexahedron if there exists a $C^{2}$-diffeomorphism from the unit cube $\widehat{K}$ to $K$.

We define $\Omega_{h}$ to be a finite collection of nonoverlapping generalized hexahedra. We define vertices of $\Omega_{h}$ to be the vertices of $K \in \Omega_{h}$, we define the edges of $\Omega_{h}$ to be the edges of $K \in \Omega_{h}$, and we define the faces of $\Omega_{h}$ to be the faces of $K \in \Omega_{h}$. We assume that any face of $K \in \Omega_{h}$ is either a face of another generalized hexahedron in $\Omega_{h}$ or part of the boundary of $\Omega_{h}$.

Each face in $\Omega_{h}$ can be parametrized with a map from the reference square $\underline{\widehat{f}}:=\left\{\left(\widehat{x}_{1}, \widehat{x}_{2}, \widehat{x}_{3}\right) \in \widehat{K}: \widehat{x}_{3}=1\right\}$ into $\mathbb{R}^{3}$,

$$
\underline{\widehat{f}} \ni\left(\widehat{x}_{1}, \widehat{x}_{2}, \widehat{x}_{3}\right) \rightarrow \psi\left(\widehat{x}_{1}, \widehat{x}_{2}\right) \in \mathbb{R}^{3} .
$$

If any given face $f=G(\widehat{f})$ of a generalized hexahedron $K \in \Omega_{h}$, where the face $\widehat{f}$ admits the characterization

$$
\widehat{f}:=\widehat{v}_{\widehat{f}}+\left\{\sum_{j=1}^{2} \widehat{x}_{\sigma(j)} e_{\sigma(j)}: \widehat{x}_{\sigma(j)} \in[0,1] \text { for } j=1,2\right\},
$$

where $\sigma:\{1,2\} \mapsto\{1,2,3\}$ is a monotone increasing injective function, we say that $\phi$ is a local orientation if it is the affine mapping from a face $\widehat{f}$ of $\widehat{K}$ onto the reference square $\underline{\hat{f}}$ such that

$$
\phi\left(\widehat{x}_{\sigma(1)}, \widehat{x}_{\sigma(2)}\right)=\left(\widehat{x}_{1}, \widehat{x}_{2}\right) .
$$


Definition 2.8. $\Omega_{h}$ is called a $C^{0}$-compatible mesh if, for any face $f$ and any generalized hexahedron $K$ which contains $f$ as a face, there is a local orientation $\phi: \widehat{f} \rightarrow \widehat{f}$ of $f$ satisfying

$$
\left.G\right|_{\widehat{f}}\left(\widehat{v}_{\widehat{f}}+\widehat{x}_{\sigma(1)} e_{\sigma(1)}+\widehat{x}_{\sigma(2)} e_{\sigma(2)}\right)=\psi\left(\phi\left(\widehat{x}_{\sigma(1)}, \widehat{x}_{\sigma(2)}\right)\right) .
$$

We define the reference interval to be $\underline{\widehat{e}}=\left\{\left(\widehat{x}_{1}, \widehat{x}_{2}, \widehat{x}_{3}\right) \in \widehat{K}: \widehat{x}_{2}=\widehat{x}_{3}=0\right\}$. Every edge in $\Omega_{h}$ can be parametrized with a map from the reference interval $\underline{\hat{e}}$ into $\mathbb{R}^{3}$ :

$$
\underline{\widehat{e}} \ni\left(\widehat{x}_{1}, \widehat{x}_{2}, \widehat{x}_{3}\right) \rightarrow \chi\left(\widehat{x}_{1}\right) \in \mathbb{R}^{3} .
$$

2.2.2. Definition of the spaces. Let $\Omega$ be an open subset in $\mathbb{R}^{3}$. Let $\Omega_{h}$ be a $C^{0}$ compatible mesh such that $\bar{\Omega}=\bigcup_{K \in \Omega_{h}} \bar{K}$. For any $K \in \Omega_{h}$, we denote by $G_{K}$ a $C^{2}$-diffeomorphism between the reference cube $\widehat{K}$ and $K$, and set $A:=D G_{K}$ and $J:=\operatorname{det} A$.

Definition 2.9. We define the finite element spaces $W_{h}, V_{h}, E_{h}$ and $H_{h}$ as the subspaces of $L^{2}(\Omega), H(\operatorname{div}, \Omega), H(\operatorname{curl}, \Omega), H^{1}(\Omega)$, respectively, such that, for any generalized hexahedron $K \in \Omega_{h}$,

$$
\begin{aligned}
\left.W_{h}\right|_{K} & :=J^{-1} W(\widehat{K}) \circ G_{K}^{-1}, \\
\left.V_{h}\right|_{K} & :=J^{-1} A V(\widehat{K}) \circ G_{K}^{-1}, \\
\left.E_{h}\right|_{K} & :=A^{-\top} E(\widehat{K}) \circ G_{K}^{-1}, \\
\left.H_{h}\right|_{K} & :=H(\widehat{K}) \circ G_{K}^{-1},
\end{aligned}
$$

where the spaces $W(\widehat{K}), V(\widehat{K}), E(\widehat{K})$ and $H(\widehat{K})$ on $\widehat{K}$ are given by Definition 2.1 . 2.2.3. Definition of the projections. Next, we define the projection operators $\Pi_{W_{h}}$, $\Pi_{V_{h}}, \Pi_{E_{h}}$, and $\Pi_{H_{h}}$.

Definition 2.10. Let $w \in L^{2}(\Omega), q \in H^{r-1}(\operatorname{div}, \Omega), \omega \in H^{r-1}(\operatorname{curl}, \Omega), u \in H^{r}(\Omega)$ for $r>3 / 2$, and let $K \in \Omega_{h}$. We set

$$
\begin{aligned}
& w\left(x_{1}, x_{2}, x_{3}\right):=J^{-1} \widehat{w}\left(\widehat{x}_{1}, \widehat{x}_{2}, \widehat{x}_{3}\right), \\
& q\left(x_{1}, x_{2}, x_{3}\right):=J^{-1} A \widehat{q}\left(\widehat{x}_{1}, \widehat{x}_{2}, \widehat{x}_{3}\right), \\
& \omega\left(x_{1}, x_{2}, x_{3}\right):=A^{-\top} \widehat{\omega}\left(\widehat{x}_{1}, \widehat{x}_{2}, \widehat{x}_{3}\right), \\
& u\left(x_{1}, x_{2}, x_{3}\right):=\widehat{u}\left(\widehat{x}_{1}, \widehat{x}_{2}, \widehat{x}_{3}\right) .
\end{aligned}
$$

Then, we define

$$
\begin{aligned}
& \Pi_{W_{h}} w\left(x_{1}, x_{2}, x_{3}\right):=J^{-1} \Pi_{W} \widehat{w}\left(\widehat{x}_{1}, \widehat{x}_{2}, \widehat{x}_{3}\right), \\
& \Pi_{V_{h}} q\left(x_{1}, x_{2}, x_{3}\right):=J^{-1} A \Pi_{V} \widehat{q}\left(\widehat{x}_{1}, \widehat{x}_{2}, \widehat{x}_{3}\right), \\
& \Pi_{E_{h}} \omega\left(x_{1}, x_{2}, x_{3}\right):=A^{-\top} \Pi_{E} \widehat{\omega}\left(\widehat{x}_{1}, \widehat{x}_{2}, \widehat{x}_{3}\right), \\
& \Pi_{H_{h}} u\left(x_{1}, x_{2}, x_{3}\right):=\Pi_{H} \widehat{u}\left(\widehat{x}_{1}, \widehat{x}_{2}, \widehat{x}_{3}\right),
\end{aligned}
$$

where $\Pi_{W}, \Pi_{V}, \Pi_{E}, \Pi_{H}$ are the projection operators on the reference cube $\widehat{K}$ given by Definitions 2.2, 2.3, 2.4 and 2.5, respectively. 
2.2.4. The commuting diagrams. We are now ready to state our main result.

Theorem 2.11. For any $k \geq 1$ and $r>3 / 2$, the operators $\Pi_{V_{h}}, \Pi_{E_{h}}$ and $\Pi_{H_{h}}$ are well-defined projections. Moreover, we have that

$$
\begin{aligned}
\nabla \cdot \Pi_{V_{h}} q & =\Pi_{W_{h}} \nabla \cdot q & & \forall q \in H^{r-1}(\operatorname{div}, \Omega), \\
\nabla \times \Pi_{E_{h}} \omega & =\Pi_{V_{h}} \nabla \times \omega & & \forall \omega \in H^{r-1}(\mathrm{curl}, \Omega), \\
\nabla \Pi_{H_{h}} u & =\Pi_{H_{h}} \nabla u & & \forall u \in H^{r}(\Omega) .
\end{aligned}
$$

\section{Proofs of the Results Associated to the Reference ElEMEnt}

This section is devoted to proving the commutativity properties of Theorem 2.6.

3.1. Preliminaries. We begin by establishing three results that provide very useful characterizations of some of the spaces employed to define the projections $\Pi_{V}$ and $\Pi_{E}$.

Spaces associated to the projection $\Pi_{V}$. We begin by considering a space used to define the projection $\Pi_{V}$.

Lemma 3.1 (Characterization of $\left.\nabla \operatorname{Ker}\left(\left.t r\right|_{Q_{k}(K)}\right)\right)$. We have that

$$
\operatorname{Ker}\left(\left.\operatorname{tr}_{t}\right|_{\left[Q_{k}(K)\right]^{3}}\right) \cap \operatorname{Ker}\left(\nabla \times\left.\right|_{\left[Q_{k}(K)\right]^{3}}\right)=\nabla \operatorname{Ker}\left(\left.\operatorname{tr}\right|_{Q_{k}(K)}\right) \text {. }
$$

Proof. Let us show that

$$
\operatorname{Ker}\left(\left.\operatorname{tr}_{t}\right|_{\left.Q_{k}(K)\right]^{3}}\right) \cap \operatorname{Ker}\left(\nabla \times\left.\right|_{\left[Q_{k}(K)\right]^{3}}\right) \supset \nabla \operatorname{Ker}\left(\left.\operatorname{tr}\right|_{Q_{k}(K)}\right) .
$$

If $\omega$ lies on $\nabla \operatorname{Ker}\left(\left.\operatorname{tr}\right|_{Q_{k}(K)}\right)$, there is a function $u \in Q_{k}(K)$ with zero trace such that $\omega=\nabla u$. Clearly, $\omega$ belongs to $\left[Q_{k}(K)\right]^{3}$. Moreover, $n \times \omega=(n \times \nabla) u=0$ on the face $f \in \mathcal{F}(K)$ since $u$ has zero trace. This proves the inclusion.

Now let us prove that

$$
\operatorname{Ker}\left(\left.\operatorname{tr}_{t}\right|_{\left[Q_{k}(K)\right]^{3}}\right) \cap \operatorname{Ker}\left(\nabla \times\left.\right|_{\left[Q_{k}(K)\right]^{3}}\right) \subset \nabla \operatorname{Ker}\left(\left.\operatorname{tr}\right|_{Q_{k}(K)}\right) .
$$

Note that, if $\omega$ belongs to $\operatorname{Ker}\left(\left.\operatorname{tr}_{t}\right|_{\left[Q_{k}(K)\right]^{3}}\right) \cap \operatorname{Ker}\left(\nabla \times\left.\right|_{\left[Q_{k}(K)\right]^{3}}\right)$, there is a function $\phi$ with zero trace such that $\omega=\nabla \phi \in\left[Q_{k}(K)\right]^{3}$. The last condition immediately implies that $\phi \in \operatorname{Ker}\left(\left.\operatorname{tr}\right|_{Q_{k+1}(K)}\right)$. It is easy to see that if $\phi \in \operatorname{Ker}\left(\left.\operatorname{tr}\right|_{Q_{k+1}(K)}\right) \backslash$ $Q_{k}(K)$, then $\nabla \phi \notin\left[Q_{k}(K)\right]^{3}$. So, $\phi \in \operatorname{Ker}\left(\left.\operatorname{tr}\right|_{Q_{k}(K)}\right)$.

This completes the proof.

Lemma 3.2 (Characterization of $\left.\nabla \times \operatorname{Ker}\left(\left.\operatorname{tr}_{t}\right|_{\left[Q_{k}(K)\right]^{3}}\right)\right)$. We have that

$$
\operatorname{Ker}\left(\left.t r_{n}\right|_{\left[Q_{k}(K)\right]^{3}}\right) \cap \operatorname{Ker}\left(\left.\nabla \cdot\right|_{\left[Q_{k}(K)\right]^{3}}\right)=\nabla \times \operatorname{Ker}\left(\left.t r_{t}\right|_{\left[Q_{k}(K)\right]^{3}}\right) .
$$

Proof. Let us show that

$$
\operatorname{Ker}\left(\left.t r_{n}\right|_{\left[Q_{k}(K)\right]^{3}}\right) \cap \operatorname{Ker}\left(\left.\nabla \cdot\right|_{\left[Q_{k}(K)\right]^{3}}\right) \supset \nabla \times \operatorname{Ker}\left(\left.t r_{t}\right|_{\left[Q_{k}(K)\right]^{3}}\right) .
$$

If $\omega$ lies in $\nabla \times \operatorname{Ker}\left(\left.\operatorname{tr}_{t}\right|_{\left[Q_{k}(K)\right]^{3}}\right)$, there is a function $v$ in $\left[Q_{k}(K)\right]^{3}$ with zero tangential trace such that $\omega=\nabla \times v$. Clearly, $\omega$ belongs to $\operatorname{Ker}\left(\left.\nabla \cdot\right|_{\left[Q_{k}(K)\right]^{3}}\right)$. That $\omega$ also belongs to $\operatorname{Ker}\left(\left.\operatorname{tr}_{n}\right|_{\left[Q_{k}(K)\right]^{3}}\right)$ follows from the fact that, on the face $f$,

$$
n_{f} \cdot \omega=n_{f} \cdot \nabla \times v=\nabla_{f} \cdot\left(v \times n_{f}\right)=0,
$$

since $v \times n_{f}=0$ on the face $f$.

Let us now show that

$$
\operatorname{Ker}\left(\left.\operatorname{tr}_{n}\right|_{\left[Q_{k}(K)\right]^{3}}\right) \cap \operatorname{Ker}\left(\left.\nabla \cdot\right|_{\left[Q_{k}(K)\right]^{3}}\right)=\nabla \times \operatorname{Ker}\left(\left.t r_{t}\right|_{\left[Q_{k}(K)\right]^{3}}\right) .
$$


By Lemma 3.5 we immediately have that

$$
\operatorname{dim} \nabla \cdot \operatorname{Ker}\left(\left.t r_{n}\right|_{\left[Q_{k}(K)\right]^{3}}\right)=\operatorname{dim} Q_{k}(K)-8=(k+1)^{3}-8 .
$$

It is obvious that $\operatorname{dim} \operatorname{Ker}\left(\left.\operatorname{tr}_{n}\right|_{\left[Q_{k}(K)\right]^{3}}\right)=3(k+1)^{2}(k-1)$. This implies that

$$
\begin{aligned}
& \operatorname{dim} \operatorname{Ker}\left(\left.\operatorname{tr}_{n}\right|_{\left[Q_{k}(K)\right]^{3}}\right) \cap \operatorname{Ker}\left(\left.\nabla \cdot\right|_{\left[Q_{k}(K)\right]^{3}}\right) \\
= & \operatorname{dim} \operatorname{Ker}\left(\left.t r_{n}\right|_{\left[Q_{k}(K)\right]^{3}}\right)-\operatorname{dim} \nabla \cdot \operatorname{Ker}\left(\left.t r_{n}\right|_{\left[Q_{k}(K)\right]^{3}}\right)=2 k^{3}-6 k+4 .
\end{aligned}
$$

So, we have that

$$
\begin{aligned}
& 2 k^{3}-6 k+4 \\
= & \operatorname{dim} \operatorname{Ker}\left(\left.\operatorname{tr}_{n}\right|_{\left[Q_{k}(K)\right]^{3}}\right) \cap \operatorname{Ker}\left(\left.\nabla \cdot\right|_{\left[Q_{k}(K)\right]^{3}}\right) \geq \operatorname{dim} \nabla \times \operatorname{Ker}\left(\left.\operatorname{tr}_{t}\right|_{\left[Q_{k}(K)\right]^{3}}\right) \\
= & \operatorname{dim} \operatorname{Ker}\left(\left.\operatorname{tr}_{t}\right|_{\left[Q_{k}(K)\right]^{3}}\right)-\operatorname{dim} \operatorname{Ker}\left(\left.\operatorname{tr}_{t}\right|_{\left[Q_{k}(K)\right]^{3}}\right) \cap \operatorname{Ker}\left(\nabla \times\left.\right|_{\left[Q_{k}(K)\right]^{3}}\right) \\
= & \operatorname{dim} \operatorname{Ker}\left(\left.\operatorname{tr}_{t}\right|_{\left[_{k}(K)\right]^{3}}\right)-\operatorname{dim} \nabla \operatorname{Ker}\left(\left.\operatorname{tr}\right|_{Q_{k}(K)}\right) \quad \text { by Lemma } \underline{3.1} \\
= & \operatorname{dim} \operatorname{Ker}\left(\left.\operatorname{tr}_{t}\right|_{\left[_{k}(K)\right]^{3}}\right)-\operatorname{dim} \operatorname{Ker}\left(\left.\operatorname{tr}\right|_{Q_{k}(K)}\right) \\
= & 3(k+1)(k-1)^{2}-(k-1)^{3}=2 k^{3}-6 k+4 .
\end{aligned}
$$

Then, $\operatorname{dim} \operatorname{Ker}\left(\left.t r_{n}\right|_{\left[Q_{k}(K)\right]^{3}}\right) \cap \operatorname{Ker}\left(\left.\nabla \cdot\right|_{\left[Q_{k}(K)\right]^{3}}\right)=\operatorname{dim} \nabla \times \operatorname{Ker}\left(\left.t r_{t}\right|_{\left[Q_{k}(K)\right]^{3}}\right)$.

This completes the proof of Lemma 3.2 .

Spaces associated to the projection $\Pi_{E}$. Now we consider two of the spaces used to define the projection $\Pi_{E}$.

Lemma 3.3 (Characterization of $\left.\nabla \times \operatorname{Ker}\left(\left.t r\right|_{Q_{k}(f)}\right)\right)$. We have that

$$
\operatorname{Ker}\left(\left.t r_{n}\right|_{\left[Q_{k}(f)\right]^{2}}\right) \cap \operatorname{Ker}\left(\left.\nabla \cdot\right|_{\left[Q_{k}(f)\right]^{2}}\right)=\nabla_{f} \times \operatorname{Ker}\left(\left.\operatorname{tr}\right|_{Q_{k}(f)}\right) .
$$

We omit the proof of this lemma as it is a two-dimensional version of the previous characterization result.

We are now ready to start proving Theorem 2.6 .

3.2. Proof of the commutativity property of $\Pi_{V}$. To prove the commutativity property of the projection $\Pi_{V}$, we proceed as follows. We first obtain a few properties of space $V(K):=\left[Q_{k}(K)\right]^{3} \oplus \delta_{V}(K)$ which are going to allow us to suitably rewrite the definition of the projection $\Pi_{V}$. We are then going to be able to prove that it is well defined and that it satisfies the commutativity property of Theorem 2.6 .

We end with a preparation for the proofs of the other two commutativity properties. We provide a generalization of this result to any dimensions, even though we are only going to need the result for the one- and two-dimensional cases.

Step 1: Properties of the space $\delta_{V}(K)$. We begin by obtaining simple but important identities relating the spaces $\delta_{V}(K),\left[Q_{k}(K)\right]^{3}$ and $Q_{k}(K)$.

We begin with the following straightforward result on the space $\delta_{V}$.

Lemma 3.4. We have that $\operatorname{tr}_{n} \delta_{V}=\{0\}$ and that

$$
\nabla \cdot \delta_{V}(K)=\operatorname{span}\left\{\tilde{P}_{\ell_{1}}\left(x_{1}\right) \tilde{P}_{\ell_{2}}\left(x_{2}\right) \tilde{P}_{\ell_{3}}\left(x_{3}\right): \ell_{i} \in\{0, k\}, i=1,2,3, \ell_{1}+\ell_{2}+\ell_{3} \neq 0\right\} .
$$

We can now state and prove the main result of this step.

Lemma 3.5. We have

$$
\begin{aligned}
& Q_{k}(K)=\nabla \cdot\left[Q_{k}(K)\right]^{3} \oplus \operatorname{span}\left\{\tilde{P}_{k}\left(x_{1}\right) \tilde{P}_{k}\left(x_{2}\right) \tilde{P}_{k}\left(x_{3}\right)\right\} \\
& Q_{k}(K)=\nabla \cdot \operatorname{Ker}\left(\left.\operatorname{tr}_{n}\right|_{\left[Q_{k}(K)\right]^{3}}\right) \oplus \operatorname{span}\{1\} \oplus \nabla \cdot \delta_{V} .
\end{aligned}
$$


Proof. The first identity is very easy to verify. To prove the second, we begin by noting that any function $v:=\left(v_{1}, v_{2}, v_{3}\right) \in\left[Q_{k}(K)\right]^{3}$ with zero normal trace of $\partial K$ is such that

$$
\begin{aligned}
& v_{1} \in \operatorname{span}\left\{\tilde{B}_{\ell_{1}}\left(x_{1}\right) \tilde{P}_{\ell_{2}}\left(x_{2}\right) \tilde{P}_{\ell_{3}}\left(x_{3}\right): \ell_{i}=0, \ldots, k, i=1,2,3 ; \ell_{1} \notin\{0,1\}\right\}, \\
& v_{2} \in \operatorname{span}\left\{\tilde{P}_{\ell_{1}}\left(x_{1}\right) \tilde{B}_{\ell_{2}}\left(x_{2}\right) \tilde{P}_{\ell_{3}}\left(x_{3}\right): \ell_{i}=0, \ldots, k, i=1,2,3 ; \ell_{2} \notin\{0,1\}\right\}, \\
& v_{3} \in \operatorname{span}\left\{\tilde{P}_{\ell_{1}}\left(x_{1}\right) \tilde{P}_{\ell_{2}}\left(x_{2}\right) \tilde{B}_{\ell_{3}}\left(x_{3}\right): \ell_{i}=0, \ldots, k, i=1,2,3 ; \ell_{3} \notin\{0,1\}\right\} .
\end{aligned}
$$

Since the derivative of $\tilde{B}_{\ell}$ is nothing but $\tilde{P}_{\ell-1}$, we get that

$$
\begin{aligned}
& \partial_{x_{1}} v_{1} \in \operatorname{span}\left\{\tilde{P}_{\ell_{1}-1}\left(x_{1}\right) \tilde{P}_{\ell_{2}}\left(x_{2}\right) \tilde{P}_{\ell_{3}}\left(x_{3}\right): \ell_{i}=0, \ldots, k, i=1,2,3 ; \ell_{1} \notin\{0,1\}\right\}, \\
& \partial_{x_{2}} v_{2} \in \operatorname{span}\left\{\tilde{P}_{\ell_{1}}\left(x_{1}\right) \tilde{P}_{\ell_{2}-1}\left(x_{2}\right) \tilde{P}_{\ell_{3}}\left(x_{3}\right): \ell_{i}=0, \ldots, k, i=1,2,3 ; \ell_{2} \notin\{0,1\}\right\}, \\
& \partial_{x_{3}} v_{3} \in \operatorname{span}\left\{\tilde{P}_{\ell_{1}}\left(x_{1}\right) \tilde{P}_{\ell_{2}}\left(x_{2}\right) \tilde{P}_{\ell_{3}-1}\left(x_{3}\right): \ell_{i}=0, \ldots, k, i=1,2,3 ; \ell_{3} \notin\{0,1\}\right\} .
\end{aligned}
$$

This readily implies that

$Q_{k}(K)=\nabla \cdot \operatorname{Ker}\left(\left.\operatorname{tr}_{n}\right|_{\left[Q_{k}(K)\right]^{3}}\right) \oplus \operatorname{span}\left\{\tilde{P}_{\ell_{1}}\left(x_{1}\right) \tilde{P}_{\ell_{2}}\left(x_{2}\right) \tilde{P}_{\ell_{3}}\left(x_{3}\right): \ell_{i} \in\{0, k\}, i=1,2,3\right\}$, and the result follows by using Lemma 3.4. This completes the proof.

Step 2: Properties of the space $V(K)$. Next we obtain three important properties of the space $V(K)$.

Lemma 3.6. The space $V(K)=[Q(K)]^{3} \oplus \delta_{V}(K)$ satisfies the following properties:

(i) $\nabla \cdot V(K)=Q_{k}(K)$,

(ii) $\operatorname{tr}_{n} V(K)=\operatorname{tr}_{n}\left[Q_{k}(K)\right]^{3}$,

(iii) $\operatorname{Ker}\left(\left.t r_{n}\right|_{V(K)}\right) \cap \operatorname{Ker}\left(\left.\nabla \cdot\right|_{V(K)}\right)=\operatorname{Ker}\left(\left.t r_{n}\right|_{\left[Q_{k}(K)\right]^{3}}\right) \cap \operatorname{Ker}\left(\left.\nabla \cdot\right|_{\left[Q_{k}(K)\right]^{3}}\right)$.

Proof. Let us prove property (i). We have

$$
\begin{aligned}
\nabla \cdot V(K) & =\nabla \cdot\left[Q_{k}(K)\right]^{3}+\nabla \cdot \delta_{V}(K) \\
& =\nabla \cdot\left[Q_{k}(K)\right]^{3} \oplus \operatorname{span}\left\{\tilde{P}_{k}\left(x_{1}\right) \tilde{P}_{k}\left(x_{2}\right) \tilde{P}_{k}\left(x_{3}\right)\right\} \quad \text { by Lemma 3.4. } \\
& =Q_{k}(K),
\end{aligned}
$$

by the first identity of Lemma 3.5. This proves property (i).

Property (ii) is satisfied thanks to Lemma 3.4

It remains to prove property (iii). We have

$$
\begin{aligned}
\Theta & :=\operatorname{Ker}\left(\left.t r_{n}\right|_{V(K)}\right) \cap \operatorname{Ker}\left(\left.\nabla \cdot\right|_{V(K)}\right) \\
& =\operatorname{Ker}\left(\left.t r_{n}\right|_{V(K)}\right) \cap \operatorname{Ker}\left(\left.\nabla \cdot\right|_{\operatorname{Ker}\left(\left.t r_{n}\right|_{V(K)}\right)}\right) \\
& =\operatorname{Ker}\left(\left.t r_{n}\right|_{V(K)}\right) \cap \operatorname{Ker}\left(\left.\nabla \cdot\right|_{\operatorname{Ker}\left(\left.t r_{n}\right|_{\left[Q_{k}(K)\right]^{3}}\right) \oplus \delta_{V}(K)}\right),
\end{aligned}
$$

by Lemma 3.4. Now, note that, by the second identity of Lemma 3.5, we have that $\nabla \cdot \operatorname{Ker}\left(\left.t r_{n}\right|_{\left[Q_{k}(K)\right]^{3}}\right) \cap \nabla \cdot \delta_{V}(K)=\{0\}$, and, since the divergence operator is injective on $\delta_{V}$, we can write

$$
\begin{aligned}
\Theta & =\operatorname{Ker}\left(\left.t r_{n}\right|_{V(K)}\right) \cap \operatorname{Ker}\left(\left.\nabla \cdot\right|_{\operatorname{Ker}\left(\left.t r_{n}\right|_{\left[Q_{k}(K)\right]^{3}}\right)}\right) \\
& =\operatorname{Ker}\left(\left.t r_{n}\right|_{\left[Q_{k}(K)\right]^{3}}\right) \cap \operatorname{Ker}\left(\left.\nabla \cdot\right|_{\operatorname{Ker}\left(\left.t r_{n}\right|_{\left[Q_{k}(K)\right]^{3}}\right)}\right) \\
& =\operatorname{Ker}\left(\left.t r_{n}\right|_{\left[Q_{k}(K)\right]^{3}}\right) \cap \operatorname{Ker}\left(\left.\nabla \cdot\right|_{\left.Q_{k}(K)\right]^{3}}\right) .
\end{aligned}
$$

This proves identity (iii) and completes the proof of the lemma. 
Step 3: The projection $\Pi_{V}$ is well defined. Armed with the above properties of the space $V(K)$, we can now begin to study the projection $\Pi_{V}$.

Lemma 3.7. The operator $\Pi_{V}: H^{r-1}(\operatorname{div}, K) \rightarrow V(K)$ is given by

$$
\begin{aligned}
\left(\left(\Pi_{V} q-q\right) \cdot n, \mu\right)_{\partial K}=0 & \forall \mu \in \operatorname{tr}_{n} V(K), \\
\left(\Pi_{V} q-q, \nabla w\right)_{K}=0 & \forall w \in \nabla \cdot V(K), \\
\left(\Pi_{V} q-q, v\right)_{K}=0 & \forall v \in \operatorname{Ker}\left(\left.t r_{n}\right|_{V(K)}\right) \cap \operatorname{Ker}\left(\left.\nabla \cdot\right|_{V(K)}\right) .
\end{aligned}
$$

Proof. The result follows directly from the the definition of $\Pi_{V}$, Definition 2.3 , by using the properties of the space $V(K)$ of Lemma 3.6 as well as the characterization of the space $\nabla \times \operatorname{Ker}\left(\left.\operatorname{tr}_{t}\right|_{\left[Q_{k}(K)\right]^{3}}\right)$ given by Lemma 3.2. This completes the proof.

Next, we show that the projection $\Pi_{V}$ is well defined.

Lemma 3.8. The projection $\Pi_{V}$ is well defined.

Proof. To prove the result, we begin by showing that the dimension of the space $V(K), \operatorname{dim} V(K)$, is equal to the number of equations defining $\Pi_{V}, N_{\Pi_{V}}$. In other words, we have to show that $\theta:=\operatorname{dim} V(K)-N_{\Pi_{V}}$ is equal to zero.

To do this, we begin by using the definition of $\Pi_{V}$ in Lemma 3.7 to obtain

$$
\begin{aligned}
\theta & =\operatorname{dim} V(K)-\operatorname{dim} t r_{n} V(K)-\operatorname{dim} \operatorname{Ker}\left(\left.t r_{n}\right|_{V(K)}\right) \cap \operatorname{Ker}\left(\left.\nabla \cdot\right|_{V(K)}\right)-\operatorname{dim} \nabla \nabla \cdot V(K) \\
& =\operatorname{dim} \operatorname{Ker}\left(\left.t_{n}\right|_{V(K)}\right)-\operatorname{dim} \operatorname{Ker}\left(\left.t r_{n}\right|_{V(K)}\right) \cap \operatorname{Ker}\left(\left.\nabla \cdot\right|_{V(K)}\right)-\operatorname{dim} \nabla \nabla \cdot V(K) \\
& =\operatorname{dim} \nabla \cdot \operatorname{Ker}\left(\left.t r_{n}\right|_{V(K)}\right)-\operatorname{dim} \nabla \nabla \cdot V(K) .
\end{aligned}
$$

Using the fact that $V(K)=\left[Q_{k}(K)\right]^{3} \oplus \delta_{V}$, we get

$$
\begin{aligned}
\theta= & \operatorname{dim} \nabla \cdot \operatorname{Ker}\left(\left.t r_{n}\right|_{\left[Q_{k}(K)\right]^{3}}\right)+\operatorname{dim} \nabla \cdot \operatorname{Ker}\left(\left.t r_{n}\right|_{\delta_{V}(K)}\right) \\
& -\operatorname{dim} \nabla \cdot \operatorname{Ker}\left(\left.\operatorname{tr}_{n}\right|_{\left[Q_{k}(K)\right]^{3}}\right) \cap \nabla \cdot \operatorname{Ker}\left(\left.t r_{n}\right|_{\delta_{V}(K)}\right)-\operatorname{dim} \nabla \nabla \cdot V(K) \\
= & \operatorname{dim} \nabla \cdot \operatorname{Ker}\left(\left.t r_{n}\right|_{\left[Q_{k}(K)\right]^{3}}\right)+\operatorname{dim} \nabla \cdot \delta_{V}(K) \\
& -\operatorname{dim} \nabla \cdot \operatorname{Ker}\left(\left.t r_{n}\right|_{\left[Q_{k}(K)\right]^{3}}\right) \cap \nabla \cdot \delta_{V}(K)-\operatorname{dim} \nabla \nabla \cdot V(K),
\end{aligned}
$$

by Lemma 3.4 .

Now, we only have to evaluate each of the four terms of the above right-hand side by using, respectively, the second identity of Lemma 3.5. Lemma 3.4, again the second identity of Lemma 3.5. and, finally, property (i) of Lemma 3.6. We get

$$
\theta=\left(\operatorname{dim} Q_{k}(K)-8\right)+(7)-(0)-\left(\operatorname{dim} Q_{k}(K)-1\right)=0 .
$$

It remains to show that, when we set $q$ equal to zero in the equations of Lemma 3.7. the only solution $\Pi_{V} q$ is the trivial one. We proceed as follows. By the first equation, we see that $\Pi_{V} q \in \operatorname{Ker}\left(\left.\operatorname{tr}_{n}\right|_{V(K)}\right)$. This implies that

$$
\left(\Pi_{V} q, \nabla w\right)_{K}=-\left(\nabla \cdot \Pi_{V} q, w\right)_{K},
$$

for all $w \in \nabla \cdot V$. Thus, by the second equation, we have that $\Pi_{V} q \in \operatorname{Ker}\left(\left.\nabla \cdot\right|_{V(K)}\right)$. Since this implies that $q \in \operatorname{Ker}\left(\left.t r_{n}\right|_{V(K)}\right) \cap \operatorname{Ker}\left(\left.\nabla \cdot\right|_{V(K)}\right)$, by the third equation, we conclude that $\Pi_{V} q=0$, as wanted. This completes the proof. 
Step 4: The commutativity of $\Pi_{V}$. Now that we have established the projection $\Pi_{V}$, we only have to show that it satisfies the commuting diagram.

Lemma 3.9. We have that

$$
\nabla \cdot \Pi_{V} q=\Pi_{W} \nabla \cdot q \quad \forall q \in H^{r-1}(\operatorname{div}, K) .
$$

Proof. By the property (i) of the space $V(K)$ in Lemma 3.6, and the definition of the projection $\Pi_{W}$, to show the above commutativity property is equivalent to showing that

$$
\left(\nabla \cdot \Pi_{V} q, w\right)=(\nabla \cdot q, w) \quad \forall q \in W(K)=Q_{k}(K) .
$$

But, for any given $w \in W(K)$, we have that

$$
\begin{aligned}
\left(\nabla \cdot \Pi_{V} q, w\right)_{K} & =\left\langle\Pi_{V} q \cdot n, w\right\rangle_{\partial K}-\left(\Pi_{V} q, \nabla w\right)_{K} \\
& =\langle q \cdot n, w\rangle_{\partial K}-(q, \nabla w)_{K} \\
& =(\nabla \cdot q, w),
\end{aligned}
$$

by the first two equations defining $\Pi_{V}$. This completes the proof.

The proof of the commutativity property of the projection $\Pi_{V}$ is now complete.

Step 5: Generalization to two and one dimensions. The argument we have presented in the previous subsection holds for the three-dimensional case $n=3$. However, it is not difficult to see that it can be extended, almost word-by-word, to the two-dimensional $(n=2)$ and to the one-dimensional $(n=1)$ cases.

To state the result, let us begin by extending the definition of the auxiliary space $D_{k}(C)$ for $n=2$ and $n=1$. So, given the point $v_{C} \in \mathbb{R}^{n}, d \leq n$, and the monotone increasing injective function $\sigma:\{1, \ldots, d\} \mapsto\{1, \ldots, n\}$, we set

$$
C:=v_{C}+\left\{\sum_{j=1}^{d} x_{\sigma(j)} e_{\sigma(j)}: x_{\sigma(j)} \in[0,1] \text { for } j=1, \ldots, d\right\},
$$

where $\left\{e_{i}\right\}_{i=1}^{n}$ is the canonical basis of $\mathbb{R}^{n}$, and define the space $D_{k}(C)$ by (3.1a) $D_{k}(C):=\operatorname{span}\left\{v_{k}\left(\sum_{j=1}^{d} \ell_{j} x_{\sigma(j)} e_{\sigma(j)}\right): \ell_{1}, \ldots, \ell_{d} \in\{0,1\}, \ell_{1}+\cdots+\ell_{d} \neq 0\right\}$.

Here, we set

$$
\begin{aligned}
v_{k}\left(x_{1}, x_{2}\right) & :=\left(\tilde{B}_{k+1}\left(x_{1}\right) \tilde{P}_{k}\left(x_{2}\right), \tilde{P}_{k}\left(x_{1}\right) \tilde{B}_{k+1}\left(x_{2}\right)\right) & & \text { for } n=2, \\
v_{k}\left(x_{1}\right) & :=\tilde{B}_{k+1}\left(x_{1}\right) & & \text { for } n=1 .
\end{aligned}
$$

Definition 3.10. We take $W(C):=Q_{k}(C)$ and $V(C):=\left[Q_{k}(C)\right]^{d} \oplus D_{k}(C)$.

The projections $\Pi_{W}$ and $\Pi_{V}$ are defined next.

Definition 3.11. The operator $\Pi_{W}: L^{2}(C) \rightarrow W(C)$ is given by

$$
\left(\Pi_{W} u-u, w\right)_{C}=0 \quad \forall w \in Q_{k}(C) .
$$

Definition 3.12. The operator $\Pi_{V}: H^{r-1}(\operatorname{div}, C) \rightarrow V(C)$ is given by

$$
\begin{aligned}
\left(\left(\Pi_{V} q-q\right) \cdot n, \mu\right)_{f}=0 & \forall \mu \in Q_{k}(f) \quad \text { for all faces } f \text { of } C, \\
\left(\Pi_{V} q-q, \nabla w\right)_{C}=0 & \forall w \in Q_{k}(C), \\
\left(\Pi_{V} q-q, v\right)_{C}=0 & \forall v \in \operatorname{Ker}\left(\left.t r_{n}\right|_{\left[Q_{k}(C)\right]^{d}}\right) \cap \operatorname{Ker}\left(\left.\nabla \cdot\right|_{\left[Q_{k}(C)\right]^{d}}\right) .
\end{aligned}
$$

With the above notation, we have the following result. 
Corollary 3.13. For $d \in\{1,2,3\}$ and for any polynomial degree $k \geq 1$, the operators $\Pi_{W}$ and $\Pi_{V}$ are well-defined projections. Moreover, we have that

$$
\Pi_{W} \nabla \cdot q=\nabla \cdot \Pi_{V} q,
$$

for all $q \in H^{r-1}(\operatorname{div}, C)$.

3.3. Proof of the commutativity property of $\Pi_{E}$. To prove the commutativity property of the projection $\Pi_{E}$, we proceed as in the previous case. Thus, we first obtain a few properties of space $E(K):=\left[Q_{k}(K)\right]^{3} \oplus \delta_{E}(K)$ which are going to allow us to suitably rewrite the definition of the projection $\Pi_{E}$. We then prove that it is well defined and that it satisfies the commutativity property of Theorem 2.6 .

Step 1: Properties of the space $\delta_{E}(K)$. We begin by obtaining several important properties of the space $\delta_{E}(K)$.

Lemma 3.14. We have

$$
\begin{aligned}
& \text { (a) } \nabla \times \delta_{E}(K) \subset V(K), \\
& \text { (b) } n_{f} \times \delta_{E}(K)=D_{k}(f) \quad \text { on the face } f \quad \forall f \in \mathcal{F}(K) \text {, } \\
& \text { (c) } \operatorname{Ker}\left(\nabla \times\left.\right|_{\delta_{E}(K)}\right)=\{0\} .
\end{aligned}
$$

Proof. In this proof, $\omega$ is a function in $\delta_{E}(K)$, that is, $\omega=\sum_{f \in \mathcal{F}(K)} \lambda_{f} n_{f} \times v_{f}$, where $v_{f} \in D_{k}(f)$ for all faces $f \in \mathcal{F}(K)$. Let us prove the inclusion (a). We have

$$
\begin{aligned}
\nabla \times \omega & =\sum_{f \in \mathcal{F}(K)} \nabla \times\left(n_{f} \times\left(\lambda_{f} v_{f}\right)\right) \\
& =\sum_{f \in \mathcal{F}(K)}\left(\left(\nabla \cdot\left(\lambda_{f} v_{f}\right)\right) n_{f}-\left(n_{f} \cdot \nabla\right)\left(\lambda_{f} v_{f}\right)\right) \\
& =\sum_{f \in \mathcal{F}(K)}\left(\nabla \lambda_{f} \cdot v_{f}+\lambda_{f}\left(\nabla \cdot v_{f}\right)\right) n_{f}-\left(\left(n_{f} \cdot \nabla \lambda_{f}\right) v_{f}+\lambda_{f}\left(n_{f} \cdot \nabla\right) v_{f}\right) \\
& =\sum_{f \in \mathcal{F}(K)}\left(\lambda_{f}\left(\nabla \cdot v_{f}\right) n_{f}-v_{f}\right),
\end{aligned}
$$

since, by the definition of $\lambda_{f}, \nabla \lambda_{f}=n_{f}$, and since, by the definition of $v_{f}, \nabla \lambda_{f} \cdot v_{f}=$ $n_{f} \cdot v_{f}=0$ and $\left(n_{f} \cdot \nabla\right) v_{f}=0$.

Since $\lambda_{f}\left(\nabla \cdot v_{f}\right) \in Q_{k}(K)$ and since $v_{f} \in D_{k}(f) \subset D_{k}(K)$, we can conclude that $\nabla \times \omega \in\left[Q_{k}(K)\right]^{3}+D_{k}(K)=V(K)$. Thus, the inclusion (a) holds.

Let us now prove the identity (b). On the face $f^{\prime}$, we have

$$
\begin{aligned}
\omega \times n_{f^{\prime}} & =\sum_{f \in \mathcal{F}(K)} \lambda_{f}\left(n_{f} \times v_{f}\right) \times n_{f^{\prime}} \\
& =\sum_{f \in \mathcal{F}(K)}\left(\lambda_{f}\left(n_{f} \cdot n_{f^{\prime}}\right) v_{f}-\lambda_{f}\left(v_{f} \cdot n_{f^{\prime}}\right) n_{f}\right) \\
& =v_{f^{\prime}} .
\end{aligned}
$$

This readily implies identity (b). It remains to justify the last step. First, assume that $f=f^{\prime}$. Then we have that $\lambda_{f}\left(n_{f} \cdot n_{f^{\prime}}\right)=1$, by definition of $\lambda_{f}$. Moreover, $\lambda_{f}\left(v_{f} \cdot n_{f^{\prime}}\right)=v_{f} \cdot n_{f}=0$, since $v_{f} \in D_{k}(f)$. Now, assume that $f \neq f^{\prime}$. Then, $\lambda_{f}\left(n_{f} \cdot n_{f^{\prime}}\right)=0$ whenever $n_{f^{\prime}}$ is not parallel to $n_{f}$, it is orthogonal to it, and when it is parallel, $\lambda_{f}=0$ on $f^{\prime}$, by definition of $\lambda_{f}$. Moreover, $v_{f} \cdot n_{f^{\prime}}=0$ on the face $f^{\prime}$ because $v \in D_{k}(f)$. 
Finally, let use verify property (c). Let us proceed by contradiction and assume that there is an $\omega \in \delta_{E}(K)$ of the form $\omega=\nabla \phi$, where $\phi$ is not a constant. Then, using the last argument, we have that, on the face $f$,

$$
v_{f}=\omega \times n_{f}=-n_{f} \times \nabla \phi,
$$

which implies that $\nabla_{f} \cdot v_{f}=0$. This means that $v_{f}=0$, by definition of $D_{k}(f)$, and, since the face $f$ was arbitrarily chosen, that $\omega=0$. This forces $\phi$ to be a constant and contradicts our assumption. This verifies property (c) and concludes the proof.

Step 2: Properties on the space $E(K)$. Next, we gather key properties of the space $E(K):=\left[Q_{k}(K)\right]^{3} \oplus \delta_{E}(K)$ in the following result.

Lemma 3.15. We have

$$
\begin{aligned}
\text { (i) } & \nabla \times E(K) \subset V(K), \\
\text { (ii) } & E(K) \times n=V(f) \quad \text { on } f \quad \forall f \in \mathcal{F}(K), \\
\text { (iii) } & \operatorname{Ker}\left(\left.\operatorname{tr}_{t}\right|_{E(K)}\right)=\operatorname{Ker}\left(\left.\operatorname{tr}_{t}\right|_{\left[Q_{k}(K)\right]^{3}}\right), \\
\text { (iv) } & \operatorname{Ker}\left(\nabla \times\left.\right|_{E(K)}\right)=\operatorname{Ker}\left(\nabla \times\left.\right|_{\left[Q_{k}(K)\right]^{3}}\right)
\end{aligned}
$$

Proof. The inclusion (i) follows from the definition of the spaces $E(K), V(K)$ and the inclusion (a) in Lemma 3.14. The property (ii) follows from property (b) in Lemma 3.14. The property (iii) follows from the property (ii). Finally, the property (iv) follows from property (c) of Lemma 3.14. This completes the proof.

Step 3: The projection $\Pi_{E}$ is well defined. Next, we use the above properties of the space $E(K)$ to suitably rewrite the projection $\Pi_{E}$.

Lemma 3.16. The operator $\Pi_{E}$ is defined by the equations

$$
\left(\Pi_{E} \omega\right) \times n_{f}=\Pi_{V(f)}\left(\omega \times n_{f}\right) \quad \text { on any face } f \in \mathcal{F}(K) \text {, }
$$

$$
\left(\nabla \times\left(\Pi_{E} \omega-\omega\right), \nabla \times v\right)_{K}=0 \quad \forall v \in \operatorname{Ker}\left(\left.\operatorname{tr}_{t}\right|_{E(K)}\right),
$$

$(\gamma) \quad\left(\Pi_{E} \omega-\omega, v\right)_{K}=0$

$$
\forall v \in \operatorname{Ker}\left(\left.t r_{t}\right|_{E(K)}\right) \cap \operatorname{Ker}\left(\nabla \times\left.\right|_{E(K)}\right) .
$$

Proof. Let us prove equation $(\alpha)$. By the property (ii) of the space $E(K)$ of Lemma 3.15. we have that the first three equations defining the tangential component of $\Pi_{E} \omega$, see Definition 2.4, are equivalent to the identity $(\alpha)$ by Corollary 3.13 with $d=2$ and $C:=f$, and by the characterization of the space $\nabla_{f} \times \operatorname{Ker}\left(\left.\operatorname{tr}\right|_{Q_{k}(f)}\right)$ given in Lemma 3.3 .

The equations $(\beta)$ and $(\gamma)$ follow directly from the last two sets of equations defining the projection $\Pi_{E}$ after using the last two properties of the space $E(K)$ of Lemma 3.15 and the characterization of the space $\nabla \operatorname{Ker}\left(\left.t r\right|_{Q_{k}(K)}\right)$ given in Lemma 3.1. This completes the proof.

We can now show the following result.

Lemma 3.17. The operator $\Pi_{E}$ is well defined.

Proof. By Lemma 3.16, we only have to show that the only solution $\Pi_{E} \omega=0$ when $\omega=0$ is the trivial solution. Let us show that this is actually the case. Set $\omega=0$. Then by property $(\alpha)$ of Lemma 3.16, we have that $t r_{t} \Pi_{E} \omega=0$. By the equation $(\beta)$, we obtain that $\nabla \times \Pi_{E} \omega=0$, and by the equation $(\gamma)$, that $\Pi_{E} \omega=0$. This completes the proof. 


\section{Step 4: The commutativity of $\Pi_{E}$.}

Lemma 3.18. We have

$$
\nabla \times \Pi_{E} \omega=\Pi_{V} \nabla \times \omega \quad \forall \omega \in H^{r-1}(\text { curl }, K) .
$$

Proof. By the property (i) of the space $E(K)$ of Lemma 3.15, and by the definition of the projection $\Pi_{V}$, see Definition 2.3. it is sufficent to show that for any $\omega \in$ $H^{r-1}(\operatorname{curl}, K)$, we have that

$$
\begin{aligned}
\left(\nabla \times\left(\Pi_{E} \omega-\omega\right) \cdot n, \mu\right)_{f}=0 & \forall \mu \in Q_{k}(f) \forall f \in \mathcal{F}(K), \\
\left(\nabla \times\left(\Pi_{E} \omega-\omega\right), \nabla w\right)_{K}=0 & \forall w \in Q_{k}(K), \\
\left(\nabla \times\left(\Pi_{E} \omega-\omega\right), v\right)_{K}=0 & \forall v \in \nabla \times \operatorname{Ker}\left(\left.\operatorname{tr}_{t}\right|_{\left[Q_{k}(K)\right]^{3}}\right) .
\end{aligned}
$$

Let us prove that the first equation is satisfied. Pick any face $f \in \mathcal{F}(K)$ and any $\mu \in Q_{k}(f)$. Then

$$
\begin{array}{rlrl}
\left(\nabla \times \Pi_{E} \omega \cdot n_{f}, \mu\right)_{f} & =\left(\nabla_{f} \cdot \Pi_{E} \omega, \mu\right)_{f} & & \text { since } \nabla_{f} \cdot:=n_{f} \cdot \nabla, \\
& =\left(\Pi_{V(f)} \nabla_{f} \cdot \omega, \mu\right)_{f} & & \text { by the identity }(\alpha) \text { of Lemma 3.16, } \\
& =\left(\nabla_{f} \cdot \omega, \mu\right)_{f} & & \text { by Definition 3.12, } \\
& =\left(\nabla \times \omega \cdot n_{f}, \mu\right)_{f} . &
\end{array}
$$

This proves the first equation.

A simple integration by parts shows that the second equation is trivially satisfied. It remains to verify the last equation. But this equation is nothing but the fourth equation defining $\Pi_{E}$. This proves the third equation and completes the proof of the lemma.

The proof of the commutativity property of the projection $\Pi_{E}$ is now complete.

3.4. Proof of the commutativity property of $\Pi_{H}$. To prove the commutativity property of the projection $\Pi_{H}$, we proceed as in the previous cases. Thus, we first obtain a few properties of space $H(K):=Q_{k}(K) \oplus \delta_{H}(K)$ which are going to allow us to suitably rewrite the definition of the projection $\Pi_{H}$. We then prove that it is well defined and that it satisfies the commutativity property of Theorem 2.6 .

Step 1: Properties of the space $\delta_{H}(K)$. We begin by gathering key properties of the space $\delta_{H}(K)$ in the following result.

Lemma 3.19. We have

$$
\begin{aligned}
& \text { (a) } \nabla \delta_{H}(K) \subset E(K), \\
& \text { (b) } \operatorname{tr} \delta_{H}(K)=D_{k}(e) \quad \text { on the edge e } \forall e \in \mathcal{E}(K) \text {, } \\
& \text { (c) } \operatorname{Ker}\left(\left.\operatorname{tr}\right|_{t r_{f} \delta_{H}(K)}\right)=\{0\} \quad \forall f \in \mathcal{F}(K) .
\end{aligned}
$$

Proof. In this proof, $u$ is a function in $\delta_{H}(K)$, that is, $u=\sum_{e \in \mathcal{E}(K)} \lambda_{e} u_{e} \cdot t_{e}$, where $u_{e} \in D_{k}(e)$ for $e \in \mathcal{E}(K)$. Let us show property (a). We have

$$
\nabla u=\sum_{e \in \mathcal{E}(K)}\left(\nabla \lambda_{e} u_{e} \cdot t_{e}+\lambda_{e} \nabla\left(u_{e} \cdot t_{e}\right)\right) .
$$

Let $e$ be any of the edges of $K$. Then, since $\lambda_{e} \nabla\left(u_{e} \cdot t_{e}\right) \in\left[Q_{k}(K)\right]^{3}$, we only have to show that $\nabla \lambda_{e} u_{e} \cdot t_{e}$ lie in $E(K)$. In fact, we are going to show that they lie in 
$\delta_{E}(K)$. Indeed, assume that the edge $e$ is common to the faces $f$ and $g$, and that $n_{f} \times n_{g}=t_{e}$. Then $\lambda_{e}=\lambda_{f} \lambda_{g}$ and so

$$
\nabla \lambda_{e}=\lambda_{g} n_{f}+\lambda_{f} n_{g}=\lambda_{g} n_{g} \times t_{e}-\lambda_{f} n_{f} \times t_{e} .
$$

This implies that

$$
\nabla \lambda_{e} u_{e} \cdot t_{e}=\lambda_{g} n_{g} \times\left(t_{e} u_{e} \cdot t_{e}\right)-\lambda_{f} n_{f} \times\left(t_{e} u_{e} \cdot t_{e}\right),
$$

and since

$$
\begin{aligned}
& \lambda_{g} n_{g} \times\left(t_{e} u_{e} \cdot t_{e}\right) \in \lambda_{g} n_{g} \times D_{k}(e) \subset \lambda_{g} n_{g} \times D_{k}(g) \subset \delta_{E}(K), \\
& \lambda_{f} n_{f} \times\left(t_{e} u_{e} \cdot t_{e}\right) \in \lambda_{f} n_{f} \times D_{k}(e) \subset \lambda_{f} n_{f} \times D_{k}(f) \subset \delta_{E}(K),
\end{aligned}
$$

we see that $\nabla \lambda_{e} u_{e} \cdot t_{e} \in \delta_{E}(K)$. This implies that property (a) holds.

Let us prove property (b). We have, on the edge $e^{\prime}$, that

$$
u=u_{e^{\prime}} \cdot t_{e^{\prime}}+\sum_{\substack{e \in \mathcal{E}(K) \\ t_{e} \cdot t_{e^{\prime}}=0}} \lambda_{e} u_{e} \cdot t_{e},
$$

by the definition of the bilinear functions $\lambda_{e}$. But, if $t_{e} \cdot t_{e^{\prime}}=0$, then $u_{e} \cdot t_{e}=0$ on $e^{\prime}$, and we get that

$$
u=u_{e^{\prime}} \cdot t_{e^{\prime}}
$$

This shows that property (b) holds.

Finally, it is not difficult to see that property (c) follows from property (ii). This completes the proof.

Step 2: Properties of the space $H(K)$. We now have the following properties of the space $H(K)$.

Lemma 3.20. We have

$$
\begin{array}{ll}
\text { (i) } & \nabla H(K) \subset E(K), \\
\text { (ii) } & \operatorname{tr} H(K)=V(e) \quad \text { on the edge e } \forall e \in \mathcal{E}(K), \\
\text { (iii) } \operatorname{Ker}\left(\left.\operatorname{tr}\right|_{t r_{f} H(K)}\right)=\operatorname{Ker}\left(\left.\operatorname{tr}\right|_{Q_{k}(f)}\right) & \forall f \in \mathcal{F}(K), \\
\text { (iv) } \operatorname{Ker}\left(\left.\operatorname{tr}\right|_{H(K)}\right)=\operatorname{Ker}\left(\left.\operatorname{tr}\right|_{Q_{k}(K)}\right) . &
\end{array}
$$

Proof. The inclusion (i) follows from the definition of the spaces $H(K), E(K)$ and the inclusion (a) in Lemma 3.19, The property (ii) follows from property (b) in Lemma 3.19. Finally, properties (iii) and (iv) follow from property (c) of Lemma 3.19. This completes the proof.

\section{Step 3: The projection $\Pi_{H}$ is well defined.}

Lemma 3.21. The projection $\Pi_{H}$ is defined by the equations

$$
\begin{array}{ll}
\Pi_{H} u=\Pi_{V(e)}(u) & \forall e \in \mathcal{E}(K), \\
\left(\Pi_{H} u-u, \Delta \zeta\right)_{f}=0 & \forall \zeta \in \operatorname{Ker}\left(\left.t r\right|_{t r_{f} H(K)}\right) \quad \forall f \in \mathcal{F}(K), \\
\left(\nabla\left(\Pi_{H} u-u\right), \nabla w\right)_{K}=0 & \forall w \in \operatorname{Ker}\left(\left.t r\right|_{H(K)}\right) .
\end{array}
$$

Proof. Let us prove equation $(\alpha)$. By property (ii) of the space $H(K)$ of Lemma 3.20. we have that the first two equations defining the trace of $\Pi_{H} u$ on the edges are equivalent to the identity $(\alpha)$ by Corollary 3.13 with $d=1$ and $C:=e$. The equations $(\beta)$ and $(\gamma)$ follow directly from the last two equations defining the projection $\Pi_{H}$ after using the last two properties of the space $H(K)$ of Lemma 3.20 , This completes the proof. 
Lemma 3.22. The projection $\Pi_{H}$ is well defined.

Proof. By Lemma 3.21, we only have to show that the only solution $\Pi_{H} u=0$ when $u=0$ is the trivial solution. So, set $u=0$. Then by property $(\alpha)$ of Lemma 3.21 we have that $\Pi_{H} u=0$ on all edges $e$ of $K$. By the equation $(\beta)$, we obtain that $\Pi_{H} u=0$ on all faces $f$ of $K$, and by the equation $(\gamma)$, that $\Pi_{H} u=0$. This completes the proof.

\section{Step 4: The commutativity of $\Pi_{H}$.}

Lemma 3.23. We have

$$
\nabla \Pi_{H} u=\Pi_{E} \nabla u \quad \forall u \in H^{r}(K) .
$$

Proof. By the property (i) of the space $H(K)$ of Lemma 3.20 , and by the definition of the projection $\Pi_{E}$, it is sufficent to show that for any $\omega \in H^{r-1}$ (curl, $K$ ), we have that

$$
\begin{aligned}
\left(\nabla\left(\Pi_{H} u-u\right) \cdot t, \mu\right)_{e}=0 & \forall \mu \in Q_{k}(e) \forall e \in \mathcal{E}(K), \\
\left(\nabla\left(\Pi_{H} u-u\right) \times n, \nabla \zeta\right)_{f}=0 & \forall \zeta \in Q_{k}(f) \quad \forall f \in \mathcal{F}(K), \\
\left(\nabla\left(\Pi_{H} u-u\right) \times n, z\right)_{f}=0 & \forall z \in \nabla_{f} \times \operatorname{Ker}\left(\left.t r\right|_{Q_{k}(f)}\right) \forall f \in \mathcal{F}(K), \\
\left(\nabla \times \nabla\left(\Pi_{H} u-u\right), \nabla \times v\right)_{K}=0 & \forall v \in \operatorname{Ker}\left(\left.\operatorname{tr}_{t}\right|_{\left[Q_{k}(K)\right]^{3}}\right), \\
\left(\nabla\left(\Pi_{H} u-u\right), v\right)_{K}=0 & \forall v \in \nabla \operatorname{Ker}\left(\left.t r\right|_{Q_{k}(K)}\right) .
\end{aligned}
$$

Let us prove that the first equation is satisfied. Pick any edge $e \in \mathcal{E}(K)$ and any $\mu \in Q_{k}(e)$. Then

$$
\begin{aligned}
\left(\nabla \times \Pi_{H} u \cdot t_{e}, \mu\right)_{e} & =\left(\nabla_{e} \Pi_{H} u, \mu\right)_{e} & & \text { where } \nabla_{e}:=t_{e} \cdot \nabla, \\
& =\left(\Pi_{V(e)} \nabla_{e} u, \mu\right)_{e} & & \text { by the identity }(\alpha) \text { of Lemma 3.21] } \\
& =\left(\nabla_{e} u, \mu\right)_{e} & & \text { by Definition 3.12, } \\
& =\left(\nabla u \cdot t_{e}, \mu\right)_{e} . & &
\end{aligned}
$$

This proves the first equation.

A simple integration by parts shows that the second equation is trivially satisfied.

For the third equation, we proceed as follows. Since it is equivalent to

$$
\left(n \times \nabla\left(\Pi_{H} u-u\right), n \times \nabla \zeta\right)_{f}=0 \quad \forall \zeta \in \operatorname{Ker}\left(\left.t r\right|_{Q_{k}(f)}\right) \forall f \in \mathcal{F}(K),
$$

we have that

$$
\begin{aligned}
\left(n \times \nabla \Pi_{H} u, n \times \nabla \zeta\right)_{f} & =\left(\nabla_{f} \times \Pi_{H} u, \nabla_{f} \times \zeta\right)_{f} \\
& =\left(\nabla_{f} \Pi_{H} u, \nabla_{f} \zeta\right)_{f} \\
& =-\left(\Pi_{H} u, \Delta \zeta\right)_{f}+\sum_{e \in \mathcal{E}(f)}\left(\Pi_{H} u, n_{e} \cdot \nabla_{f} \zeta\right)_{e} \\
& =-(u, \Delta \zeta)_{f}+\sum_{e \in \mathcal{E}(f)}\left(u, n_{e} \cdot \nabla_{f} \zeta\right)_{e},
\end{aligned}
$$

by equation $(\beta)$ of Lemma 3.21 and by the first property just proved.

The fourth equation is trivially true, and the fifth equation is nothing but the last equation defining the projection $\Pi_{H}$. This completes the proof.

This completes the proof of Theorem 2.6. 


\section{Proofs of the Results associated to the entire mesh}

This section is devoted to proving the commutativity properties of Theorem 2.11. We are only going to prove the second commutativity property since it is the most difficult of the three. The other two properties can be proven in a similar but much simpler manner. This would complete the proof of Theorem 2.11.

We proceed as follows. First, we give a characterization of the space of the tangential traces of the functions in $\left.E_{h}\right|_{K}$ in order to establish that it is isomorphic to the corresponding space in the reference element. Then, we show that the projection $\left.\Pi_{E_{h}}\right|_{K}$ satisfies a commutativity property. Finally, we show that the tangential trace of the projection $\Pi_{E_{h}}$ is single-valued in interelement boundaries. This ensures the fact that the image of $\Pi_{E_{h}}$ belongs to $E_{h}$ and completes the proof of the commutativity property of $\Pi_{E_{h}}$ in Theorem 2.11.

4.1. Characterization of the tangential traces of the functions in $\left.E_{h}\right|_{K}$. The notation we use for the next result is the following. Let $K$ be a generalized hexahedron in $\Omega_{h}$ and $f$ be one of its faces. Let $G_{K}$ the $C^{2}$-diffeomorphism mapping the reference cube $\widehat{K}$ onto $K$. We define $\widehat{f}$ be the face of reference unit cube $\widehat{K}$ such that $G_{K}(\widehat{f})=f$. Here, $n, \underline{\widehat{n}}$ are the unit normal vectors along $f, \underline{\widehat{f}}$ respectively. Finally, we let $\psi: \widehat{f} \rightarrow f$ be the parametrization of $f$.

We are now ready to state the result.

Lemma 4.1. We have that

$$
\left.\left[n \times\left(\left.E_{h}\right|_{K}\right)\right]\right|_{f}=\left\{\frac{C}{\sqrt{\operatorname{det}\left(C^{\top} C\right)}} \operatorname{tr}_{t, \underline{\hat{f}}}\left[\underline{\widehat{\widehat{x}}} \times \underline{\widehat{\omega}} \circ \psi^{-1}\right]: \forall \underline{\widehat{\omega}} \in\left\{\left[Q_{k}(\underline{\widehat{f}}) \oplus D_{k}(\underline{\widehat{f}})\right]^{2}\right\},\right.
$$

where $C:=D \psi \circ \psi^{-1} \in \mathbb{R}^{3 \times 2}$.

To prove this lemma, we are going to need the following auxiliary result. Therein, $\phi$ is the local orientation from the face $\widehat{f}$ of $\widehat{K}$ onto the reference square $\underline{\hat{f}}$, that is, it is an affine mapping such that $\phi\left(\widehat{x}_{\sigma(1)}, \widehat{x}_{\sigma(2)}\right)=\left(\widehat{x}_{1}, \widehat{x}_{2}\right)$.

Lemma 4.2. The mapping

$$
\widehat{\eta}\left(\widehat{x}_{\sigma(1)}, \widehat{x}_{\sigma(2)}\right) \mapsto D \phi \widehat{\eta}\left(\phi\left(\widehat{x}_{\sigma(1)}, \widehat{x}_{\sigma(2)}\right)\right)
$$

is an isomorphism from tr $\operatorname{ta}_{, \widehat{f}}\left\{\left[Q_{k}(\widehat{f})\right]^{2} \oplus D_{k}(\widehat{f})\right\}$ onto $\operatorname{tr}_{t, \underline{\underline{f}}}\left\{\left[Q_{k}(\underline{\widehat{f}})\right]^{2} \oplus D_{k}(\underline{\widehat{f}})\right\}$.

Proof. Since $\phi$ is an affine mapping from $\widehat{f}$ onto $\underline{\hat{f}}$, it is easy to see that the mapping defined by (4.1) is an isomorphism from $\operatorname{tr}_{t, \widehat{f}}\left[Q_{k}(\widehat{f})\right]^{2}$ onto $t r_{t, \underline{f}}\left[Q_{k}(\underline{\hat{f}})\right]^{2}$. It remains to show that it is also an isomorphism from $\operatorname{tr}_{t, \hat{f}} D_{k}(\widehat{f})$ onto $\operatorname{tr}_{t, \underline{\hat{f}}} D_{k}(\underline{\widehat{f}})$.

To do that, we begin by noting that

$$
\begin{aligned}
t r_{t, \widehat{f}} D_{k}(\widehat{f})=\operatorname{span}\{ & \left(0, \tilde{B}_{k+1}\left(\widehat{x}_{\sigma(2)}\right)\right), \quad\left(\tilde{B}_{k+1}\left(\widehat{x}_{\sigma(1)}\right), 0\right), \\
& \left.\left(\tilde{B}_{k+1}\left(\widehat{x}_{\sigma(1)}\right) \tilde{P}_{k}\left(\widehat{x}_{\sigma(2)}\right), \tilde{B}_{k+1}\left(\widehat{x}_{\sigma(2)}\right) \tilde{P}_{k}\left(\widehat{x}_{\sigma(1)}\right)\right)\right\},
\end{aligned}
$$

where $\left\{\widehat{x}_{\sigma(1)}, \widehat{x}_{\sigma(2)}\right\}$ are the coordinates on $\widehat{f}$.

Since the local orientation $\phi$ can be written as a composition of the mappings

$$
\begin{array}{ll}
\left(\widehat{x}_{\sigma(1)}, \widehat{x}_{\sigma(2)}\right) \rightarrow\left(\widehat{x}_{\sigma(1)}, \widehat{x}_{\sigma(2)}\right), & \left(\widehat{x}_{\sigma(1)}, \widehat{x}_{\sigma(2)}\right) \rightarrow\left(1-\widehat{x}_{\sigma(1)}, \widehat{x}_{\sigma(2)}\right), \\
\left(\widehat{x}_{\sigma(1)}, \widehat{x}_{\sigma(2)}\right) \rightarrow\left(\widehat{x}_{\sigma(1)}, 1-\widehat{x}_{\sigma(2)}\right), & \left(\widehat{x}_{\sigma(1)}, \widehat{x}_{\sigma(2)}\right) \rightarrow\left(\widehat{x}_{\sigma(2)}, \widehat{x}_{\sigma(1)}\right),
\end{array}
$$


and since

$$
\tilde{P}_{l}(y)=(-1)^{l} \tilde{P}_{l}(1-y), \quad \tilde{B}_{k+1}(y)=(-1)^{k+1} \tilde{B}_{k+1}(1-y),
$$

it is easy to check that the mapping defined in (4.1) is an isomorphism from $t r_{t, \widehat{f}} D_{k}(\widehat{f})$ onto $t r_{t, \underline{\hat{f}}} D_{k}(\underline{\hat{f}})$. This completes the proof.

We are now ready to prove Lemma 4.1

Proof of Lemma 4.1. We recall that for any $\left.\omega \in E_{h}\right|_{K}$, there is a unique $\widehat{\omega} \in E(\widehat{K})$ such that

$$
\omega\left(x_{1}, x_{2}, x_{3}\right)=D G_{K}^{-\top} \widehat{\omega}\left(\widehat{x}_{1}, \widehat{x}_{2}, \widehat{x}_{3}\right) .
$$

Let $n$ and $\widehat{n}$ be outward unit normal vectors along $f$ and $\widehat{f}$, respectively. We recall that $\widehat{f}$ is represented as

$$
\widehat{f}:=\widehat{v}_{\widehat{f}}+\left\{\widehat{x}_{\sigma(1)} e_{\sigma(1)}+\widehat{x}_{\sigma(2)} e_{\sigma(2)}: \widehat{x}_{\sigma(1)}, \widehat{x}_{\sigma(2)} \in[0,1]\right\} .
$$

Here, $\sigma:\{1,2\} \rightarrow\{1,2,3\}$ is a monotone increasing injective function. Also, $\widehat{v}_{\widehat{f}} \in \mathbb{R}^{3}$. We write $\left.\widehat{\omega}\right|_{\widehat{f}}$ as $\widehat{\omega}\left(\widehat{x}_{\sigma(1)}, \widehat{x}_{\sigma(2)}\right)$. By simple calculation, we have that for any $\left(x_{1}, x_{2}, x_{3}\right)=G_{K}\left(\widehat{v}_{\widehat{f}}+\widehat{x}_{\sigma(1)} e_{\sigma(1)}+\widehat{x}_{\sigma(2)} e_{\sigma(2)}\right)$,

$$
\begin{aligned}
\omega\left(x_{1}, x_{2}, x_{3}\right) \times n & =\frac{\left[\operatorname{det} D G_{K}\right]^{-1} D G_{K}}{\left\|D G_{K}^{-\top} \widehat{n}\right\|}\left(\widehat{x}_{\sigma(1)}, \widehat{x}_{\sigma(2)}\right) \times \widehat{n} \\
& =\frac{D G_{K}}{\sqrt{\operatorname{det}\left(\left.\left.D G_{K}^{\top}\right|_{\widehat{f}} D G_{K}\right|_{\widehat{f}}\right)}} \widehat{\omega}\left(\widehat{x}_{\sigma(1)}, \widehat{x}_{\sigma(2)}\right) \times \widehat{n} \\
& \left.=\frac{\left.D G_{K}\right|_{\widehat{f}}}{\sqrt{\operatorname{det}\left(\left.\left.D G_{K}^{\top}\right|_{\widehat{f}} D G_{K}\right|_{\widehat{f}}\right)}} t r_{t, \widehat{f}} \widehat{\omega}\left(\widehat{x}_{\sigma(1)}, \widehat{x}_{\sigma(2)}\right) \times \widehat{n}\right] .
\end{aligned}
$$

Since $\Omega_{h}$ is $C^{0}$-compatible, we have that

$$
\left.G_{K}\right|_{\widehat{f}}\left(\widehat{v}_{\widehat{f}}+\widehat{x}_{\sigma(1)} e_{\sigma(1)}+\widehat{x}_{\sigma(2)} e_{\sigma(2)}\right)=\psi\left(\phi\left(\widehat{x}_{\sigma(1)}, \widehat{x}_{\sigma(2)}\right)\right) .
$$

This implies that

$$
\left.D G_{K}\right|_{\widehat{f}}=D \psi D \phi .
$$

Since $\phi$ is an affine diffeomorphism from $\widehat{f}$ onto $\underline{\hat{f}}$, we have that $(D \phi)^{\top} D \phi=I_{2}$. This implies that

$$
\operatorname{det}\left(\left.\left.D G_{K}^{\top}\right|_{\widehat{f}} D G_{K}\right|_{\widehat{f}}\right)\left(\widehat{v}_{\widehat{f}}+\widehat{x}_{\sigma(1)} e_{\sigma(1)}+\widehat{x}_{\sigma(2)} e_{\sigma(2)}\right)=\operatorname{det}\left(C^{\top} C\right)\left(\phi\left(\widehat{x}_{\sigma(1)}, \widehat{x}_{\sigma(2)}\right)\right) .
$$

Finally, since by Definition 2.1 of $E(\widehat{K})$, we have that

$$
\widehat{n} \times\left. E(\widehat{K})\right|_{\widehat{f}}=\left[Q_{k}(\widehat{f})\right]^{2}+D_{k}(\widehat{f}),
$$

by Lemma 4.2, the proof is complete.

4.2. The local commutativity property. We begin by proving that the commutativity property we want to prove does hold inside each of the generalized hexahedra of the triangulation $\Omega_{h}$.

Lemma 4.3. Let $K$ be any generalized hexahedron of the triangulation $\Omega_{h}$. Then on $K$ we have that

$$
\nabla \times \Pi_{E_{h}} w=\Pi_{V_{h}} \nabla \times w .
$$


The proof of this result is fairly standard and is omitted. It follows from the corresponding commutativity property for the reference element of Theorem 2.6 and from the definition of the global projections.

4.3. The single-valuedness of the tangential trace of $\Pi_{E_{h}}$. It only remains to show that the tangential trace of $\Pi_{E_{h}}$ on each face of the triangulation $\Omega$ is actually single valued.

Lemma 4.4. The tangential trace of the projection $\Pi_{E_{h}} \omega$ is single valued on each face $f$ of each generalized hexahedron $K \in \Omega_{h}$.

To prove this lemma, we are going to use the following result. Its proof is straightforward.

Lemma 4.5. The mapping

$$
\widehat{\eta}\left(\widehat{x}_{\sigma(1)}, \widehat{x}_{\sigma(2)}\right) \mapsto D \phi^{-\top} \widehat{\eta}\left(\phi\left(\widehat{x}_{\sigma(1)}, \widehat{x}_{\sigma(2)}\right)\right)
$$

is an isomorphism from

$$
\operatorname{tr}_{t, \widehat{f}}\left\{\nabla_{\widehat{f}} Q_{k}(\widehat{f})+\operatorname{Ker}\left(\left.\operatorname{tr}_{n}\right|_{\left[Q_{k}(\widehat{f})\right]^{2}}\right) \cap \operatorname{Ker}\left(\left.\nabla_{\widehat{f}} \cdot\right|_{\left[Q_{k}(\widehat{f})\right]^{2}}\right)\right\}
$$

onto

$$
\operatorname{tr} t, \underline{\underline{\hat{f}}}\left\{\nabla_{\underline{\hat{f}}} Q_{k}(\underline{\widehat{f}})+\operatorname{Ker}\left(\left.\operatorname{tr}_{n}\right|_{\left[Q_{k}(\underline{\hat{f}})\right]^{2}}\right) \cap \operatorname{Ker}\left(\left.\nabla_{\underline{\hat{f}}} \cdot\right|_{\left[Q_{k}(\underline{\hat{f}})\right]^{2}}\right)\right\} .
$$

We are ready to prove Lemma 4.4

Proof. If we insert into the first three equations defining the projection $\Pi_{E}$ on the reference element $\widehat{K}$, the equations relating $\left.\Pi_{E_{h}}\right|_{K}$ to $\Pi_{E}$ (see Definition 2.4), we immediately obtain that

$$
\begin{array}{ll}
\left\langle\left(A^{\top}\left(\Pi_{E_{h}} \omega-\omega\right) \circ G_{K}\right) \cdot \widehat{t}, \widehat{\mu}\right\rangle_{\widehat{e}}=0, & \forall \widehat{\mu} \in Q_{k}(\widehat{e}), \\
\left\langle\left[A^{\top}\left(\Pi_{E_{h}} \omega-\omega\right) \circ G_{K}\right] \times \widehat{n}, \nabla_{\widehat{f}} \widehat{\zeta}\right\rangle_{\widehat{f}}=0, & \forall \widehat{\zeta} \in Q_{k}(\widehat{f}), \\
\left\langle\left[A^{\top}\left(\Pi_{E_{h}} \omega-\omega\right) \circ G_{K}\right] \times \widehat{n}, \widehat{z}\right\rangle_{\widehat{f}}=0, & \forall \widehat{z} \in \operatorname{Ker}\left(\left.\operatorname{tr}_{n}\right|_{\left[Q_{k}(\widehat{f})\right]^{2}}\right) \cap \operatorname{Ker}\left(\left.\nabla_{\widehat{f}} \cdot\right|_{\left[Q_{k}(\widehat{f})\right]^{2}}\right),
\end{array}
$$

where in the last equation we use the characterization of the space $\nabla_{\widehat{f}} \times \operatorname{Ker}\left(\left.\operatorname{tr}\right|_{Q_{k}(\widehat{f})}\right.$ given in Lemma 3.3. Recall that $A:=D G_{K}$, where $G_{K}$ maps the reference element $\widehat{K}$ into the generalized hexahedron $K$.

Next, we perform a simple change of variables to rewrite the integrals over $\widehat{e}$ and $\widehat{f}$ as integrals over $e:=G_{K}(\widehat{e})$ and $f:=G_{K}(\widehat{F})$. In what follows, $t:=A \widehat{t} /\|A \widehat{t}\|$ is the unit tangent to the generalized edge $e$, and $n:=A^{-\top} \widehat{n} /\left\|A^{-\top} \widehat{n}\right\|$ is the unit outward normal to the generalized face $f$. We readily obtain that

$$
\begin{array}{ll}
\left\langle\left(\Pi_{E_{h}} \omega-\omega\right) \cdot t, \widehat{\mu} \circ G_{K}^{-1}\right\rangle_{e}=0, & \forall \widehat{\mu} \in Q_{k}(\widehat{e}), \\
\left\langle A^{-1}\left[\left(\Pi_{E_{h}} \omega-\omega\right) \times n\right], \nabla_{\widehat{f}} \widehat{\zeta} \circ G_{K}^{-1}\right\rangle_{f}=0, & \forall \widehat{\zeta} \in Q_{k}(\widehat{f}), \\
\left\langle A^{-1}\left[\left(\Pi_{E_{h}} \omega-\omega\right) \times n\right], \widehat{z} \circ G_{K}^{-1}\right\rangle_{f}=0, & \forall \widehat{z} \in \operatorname{Ker}\left(\left.\operatorname{tr}_{n}\right|_{\left[Q_{k}(\widehat{f})\right]^{2}}\right) \cap \operatorname{Ker}\left(\left.\nabla_{\widehat{f}} \cdot\right|_{\left[Q_{k}(\widehat{f})\right]^{2}}\right),
\end{array}
$$

where we used that

$$
A^{\top} \omega \times \widehat{n}=\left\|A^{-\top} \widehat{n}\right\| A^{\top} \omega \times A^{\top} n=J\left\|A^{-\top} \widehat{n}\right\| A^{-1}(\omega \times n),
$$

where $J=\operatorname{det} A$, by the identity $M a \times M b=(\operatorname{det} M) M^{-\top}(a \times b)$.

Let $\widehat{f}$ and $\underline{\hat{e}}$ be the reference square and the reference interval respectively. Then $f$ and $\bar{e}$ can be parametrized by

$$
\psi: \underline{\widehat{f}} \rightarrow f, \quad \chi_{e}: \underline{\widehat{e}} \rightarrow e .
$$


Here, $\psi\left(\widehat{x}_{1}, \widehat{x}_{2}\right) \in \mathbb{R}^{3}$ and $\chi_{e}\left(\widehat{x}_{1}\right) \in \mathbb{R}^{3}$. Since $\Omega_{h}$ is a $C^{0}$-compatible mesh, there is an affine isomorphism $\phi$ from $\widehat{f}$ to $\underline{\hat{f}}$. This implies that $\left.G_{K}\right|_{\widehat{e}}=\left.\chi_{e} \circ \phi\right|_{\widehat{e}}$. Since $\left.\phi\right|_{\widehat{e}}$ is affine, we can write that

$$
\left\langle\left(\Pi_{E_{h}} \omega-\omega\right) \cdot t, \underline{\widehat{\mu}} \circ \chi_{e}^{-1}\right\rangle_{e}=0, \quad \forall \underline{\hat{\mu}} \in Q_{k}(\underline{\widehat{e}}) \quad \forall \widehat{e} \in \mathcal{E}(\widehat{f}) .
$$

and that

$$
\begin{aligned}
& \left\langle\left(\Pi_{E_{h}} \omega-\omega\right) \times n, B\left(B^{\top} B\right)^{-1} t r_{t, \widehat{f}} \nabla_{\widehat{f}} \widehat{\zeta} \circ G_{K}^{-1}\right\rangle_{f}=0, \quad \forall \widehat{\zeta} \in Q_{k}(\widehat{f}), \\
& \left\langle\left(\Pi_{E_{h}} \omega-\omega\right) \times n, B\left(B^{\top} B\right)^{-1} t r_{t, \widehat{f}} \widehat{z} \circ G_{K}^{-1}\right\rangle_{f}=0, \\
& \forall \widehat{z} \in \operatorname{Ker}\left(\left.\operatorname{tr}_{n}\right|_{\left[Q_{k}(\widehat{f})\right]^{2}}\right) \cap \operatorname{Ker}\left(\left.\nabla_{\widehat{f}}\right|_{\left[Q_{k}(\widehat{f})\right]^{2}}\right) .
\end{aligned}
$$

Here, $B=\left.D G_{K}\right|_{\widehat{f}} \circ G_{K}^{-1}$.

Finally, by Lemma 4.5, we can rewrite the last two equations as

$$
\left\langle\left(\Pi_{E_{h}} \omega-\omega\right) \times n, C\left(C^{\top} C\right)^{-1} \operatorname{tr}_{t, \underline{\hat{f}}} \nabla_{\underline{\hat{f}} \underline{\widehat{\zeta}}} \circ \psi^{-1}\right\rangle_{f}=0, \quad \forall \underline{\widehat{\zeta}} \in Q_{k}(\underline{\widehat{f}}),
$$

$$
\begin{aligned}
& \left\langle\left(\Pi_{E_{h}} \omega-\omega\right) \times n, C\left(C^{\top} C\right)^{-1} t r_{\left.t, \underline{\hat{f}} \hat{\underline{z}} \circ \psi^{-1}\right\rangle_{f}}=0,\right. \\
& \forall \underline{\underline{z}} \in \operatorname{Ker}\left(\left.\operatorname{tr}_{n}\right|_{\left[Q_{k}(\underline{\hat{f}})\right]^{2}}\right) \cap \operatorname{Ker}\left(\left.\nabla_{\underline{\hat{f}}^{f}}\right|_{\left[Q_{k}(\underline{\hat{f}})\right]^{2}}\right) .
\end{aligned}
$$

Here, $C=D \psi \circ \psi^{-1}$.

By (4.3), (4.4a), and (4.4b), we can conclude that $n \times \Pi_{E_{h}} \omega$ is single-valued on $f$. This completes the proof.

\section{Concluding REMARKS}

\subsection{Applications.}

Second order elliptic equations. Our finite element spaces $V\left(\Omega_{h}\right)$ and $W\left(\Omega_{h}\right)$ can be used to solve the standard mixed formulation for diffusion problems with the corresponding projection operator $\Pi_{V_{h}}$ and $\Pi_{W_{h}}$. By using Theorem 2.11, the stability of this mixed finite element method can be easily obtained.

A complete a priori error analysis of these methods was carried out in 7 for the case in which the meshes are Cartesian, and in 8 for the case in which the elements generalized hexahedra. Optimal convergence and even superconvergence for the scalar variable are obtained in both cases. The refined meshes of generalized hexahedra must be obtained by refining the reference element.

Vector Poisson's equation. The variational formulation of vector Poisson's equation in three space dimensions consists in looking for $\sigma \in H(\operatorname{curl}, \Omega), p \in H(\operatorname{div}, \Omega)$ such that

$$
\begin{array}{ll}
(\sigma, \tau)-(p, \nabla \times \tau)=0 & \forall \tau \in H(\operatorname{curl}, \Omega), \\
(\nabla \times \sigma, q)+\left(\nabla \cdot p_{h}, \nabla \cdot q\right)=(f, q) & \forall q \in H(\operatorname{div}, \Omega) .
\end{array}
$$

We can set up the corresponding discrete problem by using our finite element spaces $E_{h}$ and $V_{h}$. Indeed, the corresponding discretization consists in finding $\sigma_{h} \in E_{h}, p_{h} \in V_{h}$ such that

$$
\begin{array}{ll}
\left(\sigma_{h}, \tau\right)-\left(p_{h}, \nabla \times \tau\right)=0 & \forall \tau \in E_{h}, \\
\left(\nabla \times \sigma_{h}, q\right)+\left(\nabla \cdot p_{h} \nabla \cdot q\right)=(f, q) & \forall q \in V_{h} .
\end{array}
$$


By utilizing the commutativity property of Theorem 2.11 we can easily verify that Lemma 9.1 and Theorem 9.2 in [9] hold in this case. As a consequence, the discrete problem just introduced is stable. The a priori error analysis of these methods remains to be carried out.

5.2. On the dimension of the spaces $\delta_{V}(K), \delta_{E}(K)$ and $\delta_{H}(K)$. Here, we describe in what sense the dimension of the spaces $\delta_{V}(K), \delta_{E}(K)$ and $\delta_{H}(K)$ is in fact the smallest possible.

The dimension of $\delta_{V}(K)$. We begin with the following result. We will use it, not only to establish that the dimension of $\delta_{V}(K)$ is the smallest possible, but to show the same result for $\delta_{E}(K)$ and $\delta_{H}(K)$.

Lemma 5.1. Let $\delta$ be any finite-dimensional space such that

$$
\nabla \cdot V=Q_{k}(K), \quad \text { where } \quad V:=\left[Q_{k}(K)\right]^{d} \oplus \delta .
$$

Let $\Pi_{V}: H^{r-1}($ div,$K) \rightarrow V(K)$ be any projection satisfying the equations

$$
\begin{aligned}
\left(\left(\Pi_{V} q-q\right) \cdot n, \mu\right)_{\partial K}=0 & \forall \mu \in \operatorname{tr}_{n} V(K), \\
\left(\Pi_{V} q-q, \nabla w\right)_{K}=0 & \forall w \in \nabla \cdot V(K), \\
\left(\Pi_{V} q-q, v\right)_{K}=0 & \forall v \in \operatorname{Ker}\left(\left.t r_{n}\right|_{V(K)}\right) \cap \operatorname{Ker}\left(\left.\nabla \cdot\right|_{V(K)}\right) .
\end{aligned}
$$

Then, for $\Pi_{V}$ to be well defined, we must have that

$$
\operatorname{dim} \delta \geq 2^{d}-1
$$

This implies that the space $\delta_{V}(\widehat{K})$ has the smallest possible dimension, namely, $7=2^{3}-1$.

Proof. Set $\theta=\operatorname{dim} V-N_{\Pi_{V}}$. Then, proceeding exactly as in the first lines of the proof of Lemma 3.7, we readily obtain that

$$
\begin{aligned}
\theta & =\operatorname{dim} \nabla \cdot \operatorname{Ker}\left(\left.t r_{n}\right|_{V}\right)-\operatorname{dim} \nabla \nabla \cdot V \\
& =\operatorname{dim} \nabla \cdot \operatorname{Ker}\left(\left.t r_{n}\right|_{V}\right)-\left(\operatorname{dim} Q_{k}(K)-1\right),
\end{aligned}
$$

since we are assuming that $\nabla \cdot V=Q_{k}(K)$. Now

$$
\begin{aligned}
\theta= & \operatorname{dim} \nabla \cdot \operatorname{Ker}\left(\left.\operatorname{tr}_{n}\right|_{\left[Q_{k}(K)\right]^{d}}\right)+\operatorname{dim} \nabla \cdot \operatorname{Ker}\left(\left.t r_{n}\right|_{\delta_{V}}\right) \\
& -\operatorname{dim} \nabla \cdot \operatorname{Ker}\left(\left.t r_{n}\right|_{\left[Q_{k}(K)\right]^{d}}\right) \cap \nabla \cdot \operatorname{Ker}\left(\left.t r_{n}\right|_{\delta}\right)-\left(\operatorname{dim} Q_{k}(K)-1\right),
\end{aligned}
$$

and by the second identity of Lemma 3.5

$$
\begin{aligned}
\theta= & \left(\operatorname{dim} Q_{k}(K)-2^{d}\right)+\operatorname{dim} \nabla \cdot \operatorname{Ker}\left(\left.t r_{n}\right|_{\delta}\right) \\
& -\operatorname{dim} \nabla \cdot \operatorname{Ker}\left(\left.t r_{n}\right|_{\left[Q_{k}(K)\right]^{d}}\right) \cap \nabla \cdot \operatorname{Ker}\left(\left.t r_{n}\right|_{\delta}\right)-\operatorname{dim} Q_{k}(K)+1 \\
= & \operatorname{dim} \nabla \cdot \operatorname{Ker}\left(\left.t r_{n}\right|_{\delta}\right)-\operatorname{dim} \nabla \cdot \operatorname{Ker}\left(\left.t r_{n}\right|_{\left[Q_{k}(K)\right]^{d}}\right) \cap \nabla \cdot \operatorname{Ker}\left(\left.t r_{n}\right|_{\delta}\right)-2^{d}+1 .
\end{aligned}
$$

For the projection $\Pi_{V}$ to be well defined, we must have that $\theta=\operatorname{dim} V-N_{\Pi_{V}} \geq 0$, and this happens if and only if

$$
\operatorname{dim} \nabla \cdot \operatorname{Ker}\left(\left.t r_{n}\right|_{\delta}\right) \geq \operatorname{dim} \nabla \cdot \operatorname{Ker}\left(\left.t r_{n}\right|_{\left[Q_{k}(K)\right]^{d}}\right) \cap \nabla \cdot \operatorname{Ker}\left(\left.t r_{n}\right|_{\delta}\right)+2^{d}-1 \geq 2^{d}-1 .
$$

This completes the proof. 
The dimension of $\delta_{E}(K)$. We have the following result.

Lemma 5.2. Let $\delta$ be any finite-dimensional space such that

$$
\operatorname{tr}_{n, f}[\nabla \times E(K)]=Q_{k}(f) \text { for any } f \in \mathcal{F}(K), \quad \text { where } \quad E:=\left[Q_{k}(K)\right]^{3} \oplus \delta .
$$

Let $\Pi_{E}: H^{r-1}($ curl,$K) \rightarrow E(K)$ be any projection satisfying the equations

$$
\begin{aligned}
\left(\left(\Pi_{E} \omega-\omega\right) \cdot t, \mu\right)_{e}=0 & \forall \mu \in t r_{t, e} E(K) \forall e \in \mathcal{E}(K), \\
\left(\left(\Pi_{E} \omega-\omega\right) \times n, \nabla \zeta\right)_{f}=0 & \forall \zeta \in \nabla_{f} \cdot(T(f)) \quad \forall f \in \mathcal{F}(K), \\
\left(\left(\Pi_{E} \omega-\omega\right) \times n, z\right)_{f}=0 & \forall z \in \operatorname{Ker}\left(\left.t r_{n}\right|_{T(f)}\right) \cap \operatorname{Ker}\left(\left.\nabla \cdot\right|_{T(f)}\right) \forall f \in \mathcal{F}(K) .
\end{aligned}
$$

Here, $T(f)=t r_{t, f}\left[n_{f} \times E(K)\right]$ for any $f \in \mathcal{F}(K)$. Then, for $\Pi_{E}$ to be well defined, we must have that

$$
\operatorname{dim} \delta \geq 18
$$

This implies that the space $\delta_{E}(\widehat{K})$ has the smallest possible dimension, namely, $18=(6$ faces $) \times\left(2^{2}-1\right)$.

Proof. It is enough to show that for any face $f \in \mathcal{F}(K), \operatorname{dim} t r_{t, f}\left[n_{f} \times \delta\right] \geq 3$. Given any face $f \in \mathcal{F}(K)$ and letting $n$ be the outward unit vector of $f$, the three equations in the statement of this lemma can be rewritten as

$$
\begin{aligned}
\left(\left[\left(\Pi_{E} \omega-\omega\right) \times n\right] \cdot n_{e}, \mu\right)_{e}=0 & \forall \mu \in t r_{n, e} T(f) \forall e \in \mathcal{E}(f), \\
\left(\left(\Pi_{E} \omega-\omega\right) \times n, \nabla \zeta\right)_{f}=0 & \forall \zeta \in \nabla_{f} \cdot(T(f)), \\
\left(\left(\Pi_{E} \omega-\omega\right) \times n, z\right)_{f}=0 & \forall z \in \operatorname{Ker}\left(\left.t r_{n}\right|_{T(f)}\right) \cap \operatorname{Ker}\left(\left.\nabla \cdot\right|_{T(f)}\right) .
\end{aligned}
$$

Notice that $Q_{k}(f)=\operatorname{tr}_{n, f}[\nabla \times E(K)]=\nabla_{f} \cdot t r_{t, f}[n \times E(K)]=\nabla_{f} \cdot T(f)$. So, $\operatorname{tr}_{t, f}\left[n \times \Pi_{E}\right]$ needs to satisfy the same requirement as $\Pi_{V}$ in Lemma 5.1 with $d=2$. This implies that in order to make $\Pi_{E}$ well-defined, $\operatorname{dim} t r_{t, f}\left[n_{f} \times \delta\right] \geq 2^{2}-1=3$. This completes the proof.

The dimension of $\delta_{H}(K)$. We have the following result.

Lemma 5.3. Let $\delta$ be any finite-dimensional space such that

$$
t_{t, e}[\nabla H(K)]=Q_{k}(e) \text { for any edge } e \in \mathcal{E}(K), \text { where } H:=Q_{k}(K) \oplus \delta .
$$

Let $\Pi_{H}: H^{r}(K) \mapsto H(K)$ be any projection satisfying the equations

$$
\begin{aligned}
\left(\Pi_{H} u-u\right)(v)=0 & \forall v \in \mathcal{V}(K), \\
\left(\Pi_{H} u-u, \nabla_{e} \mu\right)_{e}=0 & \forall \mu \in \operatorname{Ker}\left(\left.t r_{t, e}\right|_{H(K)}\right) \quad \forall e \in \mathcal{E}(K) .
\end{aligned}
$$

Then, for $\Pi_{H}$ to be well defined, we must have that

$$
\operatorname{dim} \delta \geq 12 .
$$

This implies that the space $\delta_{H}(\widehat{K})$ has the smallest possible dimension, namely, $12=(12$ edges $) \times\left(2^{1}-1\right)$.

Proof. We only need to show that for any edge $e \in \mathcal{E}(K), \operatorname{dim} \operatorname{tr}_{t, e} \delta \geq 1$.

Notice that $Q_{k}(e)=t r_{t, e}[\nabla H(K)]=\nabla_{e} \cdot t r_{t, e}[H(K)]$ for any edge $e \in \mathcal{E}(K)$. So, $t r_{t, e} \Pi_{H}$ needs to satisfy the same requirement as $\Pi_{V}$ in Lemma 5.1] with $d=1$. This implies that in order to make $\Pi_{H}$ well-defined, $\operatorname{dim} t r_{t, e} \delta \geq 2^{1}-1=1$. This completes the proof. 
5.3. The two-dimensional case. For the sake of completeness, we end by briefly discussing the two-dimensional case. We only consider the commuting diagrams associated with the reference element, namely, the unit square denoted here by $f$. The generalization to the whole mesh is straightforward.

The commuting diagram for the two-dimensional case is of the form

$$
\begin{array}{ccccc}
H^{r-1}(\operatorname{curl}, f) & \stackrel{\nabla \times}{\longrightarrow} & H^{r-1}(\operatorname{div}, f) & \stackrel{\nabla \cdot}{\longrightarrow} & H^{r-1}(f) \\
\downarrow \Pi_{H_{h}} & & \downarrow \Pi_{V_{h}} & & \downarrow \Pi_{W_{h}} \\
H(f) & \stackrel{\nabla \times}{\longrightarrow} & V(f) & \stackrel{\nabla \cdot}{\longrightarrow} & W(f)
\end{array}
$$

where $\nabla \times:=\left(\partial_{2},-\partial_{1}\right)$. As we are going to show, the TNT spaces are such that

$$
\begin{aligned}
W(f) & =Q_{k}(f), & & \\
V(f) & =\left[Q_{k}(f) \times Q_{k}(f)\right] \oplus \delta_{V}(f), & & \operatorname{dim} \delta_{V}(f)=3, \\
H(f) & =Q_{k}(f) \oplus \delta_{H}(f), & & \operatorname{dim} \delta_{H}(f)=4 .
\end{aligned}
$$

Note that, just as for the three-dimensional case, the dimension of $\delta_{V}(f)$ is equal to the number of vertices of $f$ minus one, whereas the dimension of $\delta_{V}(f)$ is equal to the number of edges of $f$ times the number of vertices of each edge minus one.

Note also that the only other case such that the spaces contain the tensor-product spaces $Q_{k}(f)$ or $\left[Q_{k}(f) \times Q_{k}(f)\right]$ is

$$
\begin{aligned}
W(f) & =\mathcal{P}_{k, k}(f), \\
V(f) & =\mathcal{P}_{k+1, k}(f) \times \mathcal{P}_{k, k+1}(f), \\
H(f) & =\mathcal{P}_{k+1, k+1}(f),
\end{aligned}
$$

or, equivalently,

$$
\begin{aligned}
W(f) & =Q_{k}(f), & & \\
V(f) & =\left[Q_{k}(f) \times Q_{k}(f)\right] \oplus \delta_{V}(f), & & \operatorname{dim} \delta_{V}(f)=2 k+2, \\
H(f) & =Q_{k}(f) \oplus \delta_{H}(f), & & \operatorname{dim} \delta_{H}(f)=2 k+3 .
\end{aligned}
$$

We can thus see that the TNT spaces are always smaller for any $k \geq 1$.

5.3.1. Definition of the spaces. Let us now define the TNT spaces on the unit square $f$. We use the spaces $D_{k}(f)$ and $D_{k}(e)$ defined in (3.1) for the two-dimensional case $n=2$.

Definition 5.4. We take

$$
\begin{array}{rlrl}
W(f) & :=Q_{k}(f), & & \\
V(f) & :=\left[Q_{k}(f) \times Q_{k}(f)\right] \oplus \delta_{V}(f), & & \delta_{V}(f):=D_{k}(f), \\
H(f) & :=Q_{k}(f) \quad \oplus \delta_{H}(f), & \delta_{H}(f):=\bigoplus_{e \in \mathcal{E}(f)} \lambda_{e} t_{e} \cdot D_{k}(e) .
\end{array}
$$

The space $\delta_{V}(K)$. By the definition of the space $D_{k}(K)$, (3.1), we have

$$
\begin{aligned}
& \delta_{V}(f)=\operatorname{span}\{\left.v_{k}\left(x_{1}, 0\right), v_{k}\left(0, x_{2}\right), v_{k}\left(x_{1}, x_{2}\right)\right\} \\
&=\operatorname{span}\left\{\left(\tilde{B}_{k+1}\left(x_{1}\right), 0\right),\left(0, \tilde{B}_{k+1}\left(x_{2}\right)\right),\right. \\
&\left.\left(\tilde{B}_{k+1}\left(x_{1}\right) \tilde{P}_{k}\left(x_{2}\right), \tilde{P}_{k}\left(x_{1}\right) \tilde{B}_{k+1}\left(x_{2}\right)\right)\right\} .
\end{aligned}
$$


Note that each of the functions generating this space has normal trace equal to zero; see an illustration in Figure 5 .
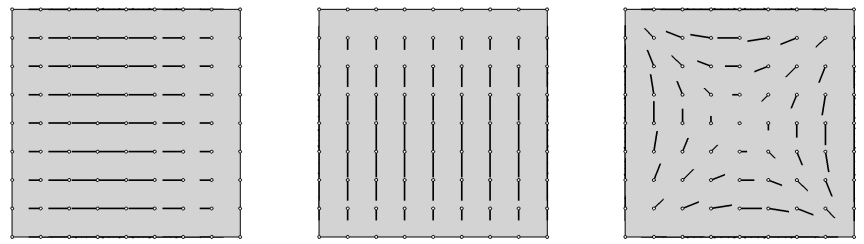

Figure 5. Trace of the bubble functions spanning the space $\delta_{V}(f)$ : The functions $v_{k}\left(x_{1}, 0\right)$ (left), $v_{k}\left(0, x_{2}\right)$ (middle), and $v_{k}\left(x_{1}, x_{2}\right)$ (right). Note that their normal trace is zero. Recall that the dimension of $\delta_{V}(f)$ is 3 . Here $k=1$.

The space $\delta_{H}(f)$. To illustrate what this space looks like, we only need to display the space $\lambda_{e} t_{e} \cdot D_{k}(e)$ for any of the edges $e$ of $f$. For example, for the edge $e$ lying on the line $x_{1}=1$, we have that $\lambda_{e}=x_{1}, t_{e}=(0,1)$ and, by the definition of the space $D_{k}(e)$, (3.1), that

$$
D_{k}(e)=\operatorname{span}\left\{v_{k}\left(0, x_{2}\right)\right\}=\operatorname{span}\left\{\left(0, \tilde{B}_{k+1}\left(x_{2}\right)\right)\right\} .
$$

As a consequence, we have

$$
\lambda_{e} t_{e} \cdot D_{k}(e)=\operatorname{span}\left\{x_{1} \tilde{B}_{k+1}\left(x_{2}\right)\right\} .
$$

Note that the trace of the function generating this space is zero except on the two faces sharing the edge $e$; see an illustration in Figure 6 .

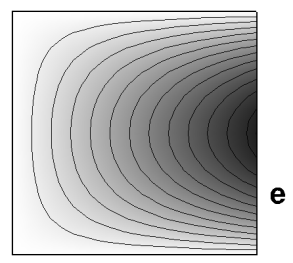

Figure 6. Trace of the bubble functions spanning the space $\delta_{H}(f)$ : The function associated to the face $e$ lying on the line $x_{1}=1$ is $\lambda_{e} t_{e} \cdot v_{k}\left(0, x_{2}\right)$. Here $\lambda_{e}=x_{1}$ and $t_{e}=(0,1)$. Note also that it is zero (white) on the faces not sharing the edge $e$. For each edge of the square, there is one of these functions so that the dimension of $\delta_{H}(K)$ is $4=(4$ edges $) \times 1$. Here $k=1$. 
5.3.2. Definition of the projections. We now define the projections as follows.

Definition 5.5. The operator $\Pi_{W}: L^{2}(f) \rightarrow W(f)$ is given by

$$
\left(\Pi_{W} u-u, w\right)_{f}=0 \quad \forall w \in Q_{k}(f) .
$$

Definition 5.6. The operator $\Pi_{V}: H^{r-1}(\operatorname{div}, f) \rightarrow V(f)$ is given by

$$
\begin{aligned}
\left(\left(\Pi_{V} q-q\right) \cdot n, \mu\right)_{e}=0 & \forall \mu \in Q_{k}(e) \forall e \in \mathcal{E}(f), \\
\left(\Pi_{V} q-q, \nabla w\right)_{f}=0 & \forall w \in Q_{k}(f), \\
\left(\Pi_{V} q-q, v\right)_{f}=0 & \forall v \in \operatorname{Ker}\left(\left.\operatorname{tr}_{n}\right|_{\left[Q_{k}(f)\right]^{2}}\right) \cap \operatorname{Ker}\left(\left.\nabla \cdot\right|_{\left[Q_{k}(f)\right]^{2}}\right) .
\end{aligned}
$$

Definition 5.7. The operator $\Pi_{H}: H^{r}(f) \rightarrow H(f)$ is given by

$$
\begin{array}{rlrl}
\left(\Pi_{H} u-u\right)(v)=0 & \forall v \in \mathcal{V}(f), & \\
\left(\Pi_{H} u-u, \nabla_{e} \mu\right)_{e}=0 & \forall \mu \in Q_{k}(e) \quad \forall e \in \mathcal{E}(f), \\
\left(\nabla\left(\Pi_{H} u-u\right), \nabla w\right)_{f}=0 & \forall w \in \operatorname{Ker}\left(\left.\operatorname{tr}\right|_{Q_{k}(f)}\right) .
\end{array}
$$

See Figure 7 for a pictorial representation of the degrees of freedom for the case $k=1$.

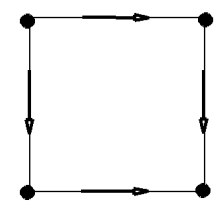

d.o.f. $\Pi_{H}=4+4=8$

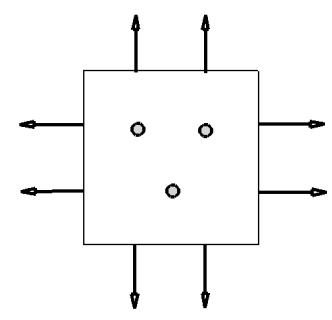

d.o.f. $\Pi_{V}=4 \times 2+3=11$

FiguRe 7. Degrees of freedom for the case $k=1: \Pi_{H}$ (left) and $\Pi_{V}$ (right).

5.3.3. The commuting diagrams. We end with the following result.

Theorem 5.8. For any polynomial degree $k \geq 1$, the operators $\Pi_{V}$ and $\Pi_{H}$ are well-defined projections. Moreover, we have that

$$
\begin{aligned}
\Pi_{W} \nabla \cdot q=\nabla \cdot \Pi_{V} q & \forall q \in H^{1}\left(f, \mathbb{R}^{3}\right), \\
\Pi_{V} \nabla \times u=\nabla \times \Pi_{H} u & \forall u \in H^{1}(f, \mathbb{R}) .
\end{aligned}
$$

Here, $\nabla \times u:=\left(\partial_{2} u,-\partial_{1} u\right)$.

We would like to end this paper by pointing out that the extension of our results to arbitrary dimensions constitutes the subject of ongoing research.

\section{ACKNOWLEDGEMENTS}

The authors would like to thank Scot Adams and an anonymous referee for constructive criticism leading to a better presentation of the material in this paper. 


\section{REFERENCES}

[1] D. N. Arnold, F. Brezzi, B. Cockburn, and L. D. Marini, Unified analysis of discontinuous Galerkin methods for elliptic problems, SIAM J. Numer. Anal. 39 (2002), 1749-1779. MR.1885715(2002k:65183)

[2] D. N. Arnold, R. Falk, and R. Winther, Finite element exterior calculus, homological techniques, and applications, Acta Numer. 15 (2006), 1-155. MR2269741 (2007j:58002)

[3] D. N. Arnold, R. Falk, and R. Winther, Finite element exterior calculus: from Hodge theory to numerical stability, Bull. Amer. Math. Soc.(N.S.) 47 (2010), 281-354. MR2594630 (2011f:58005)

[4] F. Brezzi, J. Douglas, Jr., M. Fortin, and L. D. Marini, Efficient rectangular mixed finite element methods in two and three space variables, RAIRO Modél. Math. Anal. Numér. 21 (1987), 581-604. MR921828 (88j:65249)

[5] F. Brezzi, J. Douglas, Jr., and L. D. Marini, Two families of mixed finite elements for second order elliptic problems, Numer. Math. 47 (1985), 217-235. MR799685 (87g:65133)

[6] C.D. Cantwell, S.J. Sherwin, R.M. Kirby, and P.H.J. Kelly, From $h$ to $p$ efficiency: Strategy selection for operator evaluation on hexahedral and tetrahedral elements, Computers and Fluids 43 (2011), 23-28. MR2775064

[7] B. Cockburn, W. Qiu, and K. Shi, Conditions for superconvergence of HDG methods for second-order elliptic problems, Math. Comp. 81 (2012) no. 279, 1327-1353. MR2904581

[8] _ Superconvergent HDG methods on isoparametric elements for second-order elliptic problems, SIAM J. Numer. Anal. To appear.

[9] R. Falk, P. Gatto, and P. Monk, Hexahedral $H$ (div) and $H$ (curl) finite elements, $\mathrm{M}^{2} \mathrm{AN}$ (2010). MR2781133 (2012d:65275)

[10] J.-C. Nédélec, Mixed finite elements in $\mathbf{R}^{3}$, Numer. Math. 35 (1980), 315-341. MR592160 (81k:65125)

[11] W. Qiu, and L. Demkowicz, Mixed hp-finite element method for linear elasticity with weakly imposed symmetry, Comput. Methods Appl. Mech. Engrg. 198 (2009), 3682-3701. MR2557491(2011a:74011)

[12] P. A. Raviart and J. M. Thomas, A mixed finite element method for second order elliptic problems, Mathematical Aspects of Finite Element Method, Lecture Notes in Math. 606 (I. Galligani and E. Magenes, eds.), Springer-Verlag, New York, 1977, pp. 292-315. MR0483555 $(58: 3547)$

[13] P.E.J. Vos, S.J. Sherwin, and R.M. Kirby, From $h$ to $p$ efficiency: Implementing finite and spectral/hp element methods to achieve optimal performance for low- and high-order discretizations, J. Comput. Phys. 229 (2010), 5161-5181. MR2643647 (2011i:65225)

[14] T. Warburton, L. F. Pavarino, and J. S. Hesthaven, A pseudo-spectral scheme for the incompressible Navier-Stokes equations using unstructured nodal elements, J. Comput. Phys. 164 (2000), 1-21. MR.1786240 (2001f:76062)

School of Mathematics, University of Minnesota, Minneapolis, Minnesota 55455

E-mail address: cockburn@math.umn.edu

Institute for Mathematics and Its Applications, University of Minnesota, MinneapoLIS, Minnesota 55455

E-mail address: qiuxa001@ima.umn.edu, qiuw78@gmail.com 